\title{
Minor-monotone Graph Invariants
}

\section{Alexander Schrijver}

Summary A graph parameter $\phi(G)$ is called minor-monotone if $\phi(H) \leq \phi(G)$ for any minor $H$ of $G$. We survey recent work on minor-monotone graph parameters motivated by the parameter $\mu(G)$ introduced by Colin de Verdière.

\section{Introduction}

A function $\phi(G)$ defined for any undirected graph $G$ is called minor-monotone if for any graph $G$ and any minor $H$ of $G$ one has

$$
\phi(H) \leq \phi(G) .
$$

In this paper, all graphs are undirected, loopless and without multiple edges. A minor of a graph arises by a series of deletions and contractions of edges and deletions of isolated vertices, suppressing any multiple edges and loops that may arise.

The interest in minor-monotone graph parameters is activated because the Robertson-Seymour theory of graph minors can be applied to them. Recently a number of minor-monotone parameters have been studied, motivated in particular by the graph parameter $\mu(G)$ introduced by Colin de Verdière [5] (cf. [6]). The parameter $\mu(G)$ can be described in terms of properties of matrices related to $G$. It was motivated by the study of the maximum multiplicity of the second eigenvalue of certain Schrödinger operators. When such an operator is defined on a Riemann surface, one can approximate the surface by a densely enough embedded graph $G$, in such a way that $\mu(G)$ is the maximum multiplicity of the second eigenvalue of the operator.

The interest raised by Colin de Verdière's parameter can be explained not only by its background in differential geometry, but also by the facts that it is minor-monotone (so that the Robertson-Seymour graph minors theory applies to it), and that it characterizes planarity of graphs. Indeed, one has that $\mu(G) \leq 3$ if and only if $G$ is planar. Moreover, as follows from the results in [19] and [15], $\mu(G) \leq 4$ if and only if $G$ is linklessly embeddable in $\mathbb{R}^{3}$. (A graph $G$ is linklessly embeddable if it can be embedded in $\mathbb{R}^{3}$ in such a way that the images of any two disjoint circuits in $G$ are unlinked.) So with the help of $\mu$, topological properties of a graph can be characterized in terms of spectral properties of matrices associated to the graph.

In this paper we give a survey of the graph parameter $\mu(G)$, and some related parameters, in particular the parameter $\lambda(G)$ introduced in [12]. We first give an overview of $\mu(G)$ and $\lambda(G)$, after which we give proofs of a number of results. Finally, we consider the parameters $\lambda^{\prime}(G)$ (defined by oriented matroids) and $\kappa(G)$. 
For more information we refer to the thesis by van der Holst [11], where in addition a few other minor-monotone parameters are studied.

\section{Overview of $\mu(G)$}

Let $G=(V, E)$ be an undirected graph, which we assume without loss of generality to have vertex set $V=\{1, \ldots, n\}$. Then $\mu(G)$ is the largest corank of any symmetric real-valued $n \times n$ matrix $M=\left(m_{i, j}\right)$ such that:

(i) $M$ has exactly one negative eigenvalue, of multiplicity 1 ,

(ii) for all $i, j$ with $i \neq j: m_{i, j}<0$ if $i$ and $j$ are adjacent, and $m_{i, j}=0$ if $i$ and $j$ are nonadjacent,

(iii) there is no nonzero symmetric $n \times n$ matrix $X=\left(x_{i, j}\right)$ such that $M X=0$ and such that $x_{i, j}=0$ whenever $i=j$ or $m_{i, j} \neq 0$.

There is no condition on the diagonal entries $m_{i, i}$. The corank corank $(M)$ of a matrix $M$ is the dimension of its kernel (= null space).

Note that for each graph $G=(V, E)$ a matrix $M$ satisfying (2) exists. If $G$ is connected, let $A$ be the adjacency matrix of $G$. Then we can choose $\lambda$ in such a way that $\lambda I-A$ has exactly one negative eigenvalue and is nonsingular. If $G$ is disconnected, we can choose such a $\lambda$ for each component separately and obtain again a nonsingular matrix with exactly one negative eigenvalue.

Condition (iii) is called the Strong Arnol'd Hypothesis (or Strong Arnol'd Property). There are a number of equivalent formulations of the Strong Arnol'd Hypothesis, amounting to the fact that $M$ is in a certain general position. Let $M=\left(m_{i, j}\right)$ be a symmetric $n \times n$ matrix. Let $R_{M}$ be the set of all symmetric $n \times n$ matrices $A$ with $\operatorname{rank}(A)=\operatorname{rank}(M)$. Let $S_{M}$ be the set of all symmetric $n \times n$ matrices $A=\left(a_{i, j}\right)$ such that $a_{i, j}=0$ whenever $i \neq j$ and $m_{i, j}=0$.

Then $M$ fulfils the Strong Arnol'd Hypothesis (2)(iii) if and only if

$$
R_{M} \text { intersects } S_{M} \text { at } M \text { 'transversally'; }
$$

that is, if the tangent space of $R_{M}$ at $M$ and the tangent space of $S_{M}$ at $M$ together span the space of all symmetric $n \times n$ matrices. In other words, if the intersection of the normal spaces at $M$ of $R_{M}$ and of $S_{M}$ only consists of the all-zero matrix.

It is elementary linear algebra to show that the tangent space of $R_{M}$ at $M$ consists of all symmetric $n \times n$ matrices $N$ such that $x^{T} N x=0$ for each $x \in \operatorname{ker}(M)$. Thus the normal space of $R_{M}$ at $M$ is equal to the space generated by all matrices $x x^{T}$ with $x \in \operatorname{ker}(M)$. (We assume that our underlying space is the space of real-valued symmetric $n \times n$ matrices.) This space is equal to the space of all symmetric $n \times n$ matrices $X$ satisfying $M X=0$. Trivially, the 
normal space of $S_{M}$ at $M$ consists of all symmetric $n \times n$ matrices $X=\left(x_{i, j}\right)$ such that $x_{i, j}=0$ whenever $i=j$ or $m_{i, j} \neq 0$. Therefore, (3) is equivalent to (2)(iii).

An important property of $\mu(G)$ proved by Colin de Verdière [5] is that it is monotone under taking minors:

the graph parameter $\mu(G)$ is minor-monotone.

Proving this is nontrivial, and the Strong Arnol'd Hypothesis is needed. We give the elementary proof as given in van der Holst [11] in Section 4.

The minor-monotonicity of $\mu(G)$ is especially interesting in the light of the Robertson-Seymour theory of graph minors [16], which has as principal result that if $\mathcal{C}$ is a collection of graphs so that no graph in $\mathcal{C}$ is a minor of another graph in $\mathcal{C}$, then $\mathcal{C}$ is finite. This can be equivalently formulated as follows. For any graph property $\mathcal{P}$ closed under taking minors, call a graph $G$ a forbidden minor for $\mathcal{P}$ if $G$ does not have property $\mathcal{P}$, but each proper minor of $G$ does have property $\mathcal{P}$. Note that a minor-closed property $\mathcal{P}$ is completely characterized by the collection of its forbidden minors. Now Robertson and Seymour's theorem states that each graph property that is closed under taking minors, has only finitely many forbidden minors. (See Reed's paper elsewhere in this volume.)

Since

$$
\mu\left(K_{n}\right)=n-1
$$

for each $n$ (cf. Section 5), Hadwiger's conjecture implies that $\gamma(G) \leq \mu(G)+1$ (where $\gamma(G)$ denotes the colouring number of $G$ ); this last inequality is conjectured by Colin de Verdière [5]. Since Hadwiger's conjecture holds for graphs not containing any $K_{6}$-minor (Robertson, Seymour, and Thomas [18]), we know that $\gamma(G) \leq \mu(G)+1$ holds if $\mu(G) \leq 4$.

In studying $\mu(G)$, we can restrict ourselves to considering connected graphs, since if $G$ has at least one edge, then $\mu(G)$ is equal to the maximum of $\mu(K)$ taken over all components $K$ of $G$.

The following characterizations show that with the help of $\mu(G)$, topological properties of a graph can be characterized algebraically:

(i) $\mu(G) \leq 1 \Longleftrightarrow G$ is a disjoint union of paths.

(ii) $\mu(G) \leq 2 \Longleftrightarrow G$ is outerplanar.

(iii) $\mu(G) \leq 3 \Longleftrightarrow G$ is planar.

(iv) $\mu(G) \leq 4 \Longleftrightarrow G$ is linklessly embeddable.

Here (i), (ii), and (iii) are due to Colin de Verdière [5]. In (iv), $\Longrightarrow$ is due to Robertson, Seymour, and Thomas [17] (based on the hard theorem of [19] that the Petersen family (Figure 2 on page 188) is the collection of forbidden 
minors for linkless embeddability), and $\Longleftarrow$ to Lovász and Schrijver [15]. In fact, in (6) each $\Longrightarrow$ follows from a forbidden minor characterization of the right-hand statement.

We give a proof of (i), (ii), and (iii) in Sections 9, 11, and 12, respectively. In Section 15, we indicate how $\Longleftarrow$ in (iv) can be proved, with the help of a certain Borsuk-type theorem on the existence of 'antipodal links'.

Interestingly, Kotlov, Lovász, and Vempala [14] showed that with the value $n-\mu(G)$ (for a graph $G$ with $n$ vertices) one is close to characterizing that the complementary graph $\bar{G}$ of $G$ is outerplanar or planar. In fact they showed:

$$
\begin{aligned}
& \text { if } \bar{G} \text { is a disjoint union of paths then } \mu(G) \geq n-3 \text {; } \\
& \text { if } \bar{G} \text { is outerplanar then } \mu(G) \geq n-4 \text {; } \\
& \text { if } \bar{G} \text { is planar then } \mu(G) \geq n-5 \text {. }
\end{aligned}
$$

Conversely, one has, if $G$ does not have 'twin vertices' (two (adjacent or nonadjacent) vertices $u, v$ that have the same neighbours $\neq u, v$ ), then:

$$
\begin{aligned}
& \text { if } \mu(G) \geq n-3 \text { then } \bar{G} \text { is outerplanar; } \\
& \text { if } \mu(G) \geq n-4 \text { then } \bar{G} \text { is planar. }
\end{aligned}
$$

The proof by Colin de Verdière [5] of the planarity characterization (6)(iii) uses a result of Cheng [4] on the maximum multiplicity of the second eigenvalue of Schrödinger operators defined on the sphere. A short direct proof was given by van der Holst [10], based on the following lemma. For any vector $x$, let $\operatorname{supp}(x)$ denote the support of $x$ (i.e., the set $\left\{i \mid x_{i} \neq 0\right\}$ ). Moreover, denote $\operatorname{supp}^{+}(x):=\left\{i \mid x_{i}>0\right\}$ and $\operatorname{supp}^{-}(x):=\left\{i \mid x_{i}<0\right\}$. We say that a vector $x \in \operatorname{ker}(M)$ has minimal support if $x$ is nonzero and for each nonzero vector $y \in \operatorname{ker}(M)$ with $\operatorname{supp}(y) \subseteq \operatorname{supp}(x)$ one has $\operatorname{supp}(y)=\operatorname{supp}(x)$. For any subset $U$ of $V$, let $G \mid U$ denote the subgraph of $G$ induced by $U$.

Then Van der Holst's lemma states:

Let $M$ satisfy (2) and let $x \in \operatorname{ker}(M)$ have minimal support. Then $G \mid \operatorname{supp}^{+}(x)$ and $G \mid \operatorname{supp}^{-}(x)$ are connected.

We give the proof in Section 10.

\section{Overview of $\lambda(G)$}

Van der Holst's lemma motivated van der Holst, Laurent, and Schrijver [12] to introduce a related graph parameter $\lambda(G)$, defined as follows. Let $G=(V, E)$ be a graph. Call a subspace $X$ of $\mathbb{R}^{V}$ representative for $G$ if

for each nonzero vector $x \in X, \operatorname{supp}^{+}(x)$ is nonempty and $G \mid \operatorname{supp}^{+}(x)$ is connected. 
Then $\lambda(G)$ is defined as the maximum dimension of any representative subspace $X$ of $\mathbb{R}^{V}$.

Clearly, (10) implies that also $\operatorname{supp}^{-}(x)$ is nonempty and induces a connected subgraph of $G$ for each nonzero $x \in X$.

The results characterizing $\mu$ and $\lambda$ for small values, suggest that $\lambda$ is close to $\mu$. In fact, recently Rudi Pendavingh showed that $\mu(G) \leq \lambda(G)+2$ for each graph $G$. Conversely, it might be that $\lambda(G) \leq \mu(G)$ holds.

There is a direct equivalent characterization of $\lambda(G)$. Let $G=(V, E)$ be a graph and let $d \in \mathbb{N}$. Call a function $\phi: V \rightarrow \mathbb{R}^{d}$ representative for $G$ if

for each halfspace $H$ of $\mathbb{R}^{d}$, the set $\phi^{-1}(H)$ is nonempty and induces a connected subgraph of $G$.

(Here $\phi^{-1}(H):=\{v \in V \mid \phi(v) \in H\}$.) A subset $H$ of $\mathbb{R}^{d}$ is called a halfspace if $H=\left\{x \in \mathbb{R}^{d} \mid c^{T} x>0\right\}$ for some nonzero $c \in \mathbb{R}^{d}$. Note that if $\phi: V \rightarrow \mathbb{R}^{d}$ is representative, then the vectors $\phi(v)(v \in V)$ span $\mathbb{R}^{d}$ (since otherwise there would exist a halfspace $H$ with $\left.\phi^{-1}(H)=\emptyset\right)$.

Now $\lambda(G)$ is equal to the largest $d$ for which there is a representative function $\phi: V \rightarrow \mathbb{R}^{d}$. This is easy to see. Suppose $X$ is a $d$-dimensional subspace of $\mathbb{R}^{V}$ representative for $G$. Let vectors $x_{1}, \ldots, x_{d}$ form a basis of $X$. Define $\phi(v):=\left(x_{1}(v), \ldots, x_{d}(v)\right)$ for each $v \in V$. Then $\phi$ is a representative function for $G$. Conversely, let $\phi: V \rightarrow \mathbb{R}^{d}$ be representative. Define for any $c \in \mathbb{R}^{d}$ the function $x_{c} \in \mathbb{R}^{V}$ by: $x_{c}(v):=c^{T} \phi(v)$ for $v \in V$. Then $X:=$ $\left\{x_{c} \mid c \in \mathbb{R}^{d}\right\}$ is a representative space for $G$.

It is easy to show that the function $\lambda(G)$ is minor-monotone (much easier than for $\mu(G))$ :

Theorem 3.1 If $H$ is a minor of $G$ then $\lambda(H) \leq \lambda(G)$.

Proof Let $H=\left(V^{\prime}, E^{\prime}\right)$. If $H$ arises from $G$ by deleting an isolated vertex $v_{0}$, the inequality $\lambda(H) \leq \lambda(G)$ is easy: if $\phi^{\prime}: V^{\prime} \rightarrow \mathbb{R}^{d}$ is representative for $H$ with $d=\lambda(H)$, then defining $\phi\left(v_{0}\right):=0$ and $\phi(v):=\phi^{\prime}(v)$ for all other vertices $v$ of $G$, gives a representative function for $G$.

So we may assume that $H=\left(V^{\prime}, E^{\prime}\right)$ arises from $G=(V, E)$ by deleting or contracting one edge $e=u w$. Let $\phi^{\prime}: V^{\prime} \rightarrow \mathbb{R}^{d}$ be representative for $H$ with $d=\lambda(H)$. If $H$ arises from $G$ by deleting $e$, then $V=V^{\prime}$, and $\phi^{\prime}$ is also representative for $G$. Hence $\lambda(G) \geq d=\lambda(H)$.

If $H$ arises from $G$ by contracting $e$, let $v_{0}$ be the vertex of $H$ which arose by contracting $e$. Define $\phi(u):=\phi(w):=\phi^{\prime}\left(v_{0}\right)$, and define $\phi(v):=\phi^{\prime}(v)$ for all other vertices $v$ of $G$. Then $\phi$ is representative of $G$.

One easily shows that

$$
\lambda\left(K_{n}\right)=n-1
$$

(cf. Section 5). Hence, Hadwiger's conjecture implies that $\gamma(G) \leq \lambda(G)+1$ (where $\gamma(G)$ denotes the colouring number of $G$ ). So by the truth of Hadwiger's 
conjecture for $K_{6}$-free graphs (Robertson, Seymour, and Thomas [18]), the inequality $\gamma(G) \leq \lambda(G)+1$ holds if $\lambda(G) \leq 4$.

As for the colouring number, also the function $\lambda(G)$ cannot be increased by 'clique sums'. Graph $G=(V, E)$ is a clique sum of graphs $G_{1}=\left(V_{1}, E_{1}\right)$ and $G_{2}=\left(V_{2}, E_{2}\right)$ if $V=V_{1} \cup V_{2}$ and $E=E_{1} \cup E_{2}$, where $V_{1} \cap V_{2}$ is a clique both in $G_{1}$ and in $G_{2}$. Then $\gamma(G)=\max \left\{\gamma\left(G_{1}\right), \gamma\left(G_{2}\right)\right\}$ if $G$ is a clique sum of $G_{1}$ and $G_{2}$. A similar relation holds for the size of the largest clique minor in $G$. Now in Section 6 we shall show:

If $G$ has at least one edge and is a clique sum of $G_{1}$ and $G_{2}$, then $\lambda(G)=\max \left\{\lambda\left(G_{1}\right), \lambda\left(G_{2}\right)\right\}$.

This directly gives with (12):

(i) $\lambda(G) \leq 1$ if and only if $G$ is a forest;

(ii) $\lambda(G) \leq 2$ if and only if $G$ is a series-parallel graph.

Indeed, forests can be characterized as the graphs not having a $K_{3}$-minor and also as the graphs obtainable from $K_{2}$ by taking clique sums and subgraphs. Similarly, series-parallel graphs can be characterized as the graphs not having a $K_{4}$-minor and also as the graphs obtainable from $K_{3}$ by taking clique sums and subgraphs.

In Section 13 we show that

$\lambda(G) \leq 3$ if and only if $G$ can be obtained from planar graphs by taking clique sums and subgraphs.

The kernel of the proof here is to show that $\lambda(G) \leq 3$ for any planar graph $G$. Having this, a fundamental decomposition theorem of Wagner [20] then implies the full characterization. Indeed, let $V_{8}$ be the graph with vertices $v_{1}, \ldots, v_{8}$,

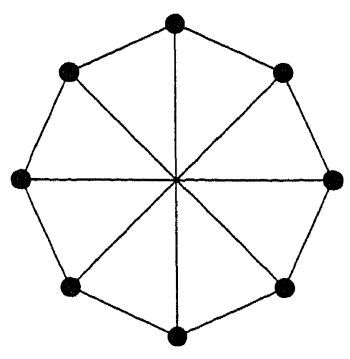

Figure 1: The graph $V_{8}$

where $v_{i}$ and $v_{j}$ are adjacent if and only if $|i-j| \in\{1,4,7\}$. Then Wagner 
showed:

$G$ can be obtained from planar graphs by taking clique sums and subgraphs $\Longleftrightarrow G$ does not have a $K_{5^{-}}$or $V_{8}$-minor.

Since $\lambda\left(K_{5}\right)=4$ and since $\lambda\left(V_{8}\right)=4$ (as we show in Section 13), we obtain (15).

In Section 16 we give a few observations concerning the class of graphs $G$ with $\lambda(G) \leq 4$. In particular, we show the result of [15] that $\lambda(G) \leq 4$ for any linklessly embeddable graph $G$. This implies with (13):

if $G$ is obtainable from linklessly embeddable graphs by taking clique sums and subgraphs, then $\lambda(G) \leq 4$.

As mentioned, an open question is if there is any direct relation between $\lambda(G)$ and $\mu(G)$. It might be the case that $\lambda(G) \leq \mu(G)$ for each graph $G$. That is, for any subspace $X$ of $\mathbb{R}^{V}$ representative for $G$ there is a matrix $M$ satisfying (2) with $\operatorname{dim}(X) \leq \operatorname{corank}(M)$. This is true if $\mu(G) \leq 4$. ber $t$ :

In fact, a tempting, more general speculation is that for any natural num-

(???) a graph $G$ satisfies $\lambda(G) \leq t$ if and only if $G$ is obtainable from graphs $H$ satisfying $\mu(H) \leq t$ by taking clique sums and subgraphs (???)

This has been proved for $t \leq 3$, and the 'if' part for $t \leq 4$.

\section{Some basic facts on $\mu(G)$}

We first prove a number of elementary facts on the parameter $\mu(G)$. We use the following notation. If $M$ is a matrix and $I$ is a set of rows of $M$ and $J$ is a set of columns of $M$, then $M_{I \times J}$ is the submatrix induced by the rows in $I$ and the columns in $J$. If $I=J$ we write $M_{I}$ for $M_{I \times I}$.

First we have the following important property due to Colin de Verdière [6], which we prove with the method described by van der Holst [11]:

Theorem 4.1 For any edge $e$ of any graph $G$ one has $\mu(G-e) \leq \mu(G)$.

Proof For any smooth manifold $\mathcal{M}$, any smooth submanifold $\mathcal{A}$ of $\mathbb{R}^{d}$, any smooth function $f: \mathcal{M} \rightarrow \mathbb{R}^{d}$, and any $x \in \mathcal{M}$ with $f(x) \in \mathcal{A}$, we say that $f$ intersects $\mathcal{A}$ transversally at $x \in \mathcal{M}$, in notation: $f \#_{x} \mathcal{A}$, if

$$
T_{f(x)} \mathcal{A}+D f_{x}\left(T_{x} \mathcal{M}\right)=\mathbb{R}^{d}
$$

Here $T_{y} \mathcal{N}$ denotes the tangent space of $\mathcal{N}$ at $y$, and $D f_{x}$ the differential of at $x$. 
A basic property of transversality is:

If $f \#_{x} \mathcal{A}$, then there is a neighbourhood $U$ of $x$ in $\mathcal{M} \cap f^{-1}(\mathcal{A})$ such that $f \#_{y} \mathcal{A}$ for each $y \in U$, and such that $U$ has the same codimension in $\mathcal{M}$ as $\mathcal{A}$ has in $\mathbb{R}^{d}$.

Let $\mathcal{S}_{n}$ denote the collection of real-valued symmetric $n \times n$ matrices, and $\mathcal{S}_{n, k}$ the collection of matrices in $\mathcal{S}_{n}$ of corank $k$. For any graph $G=(V, E)$ let $\mathcal{O}_{G}$ be the collection of real-valued symmetric $V \times V$ matrices $M$ satisfying (2)(ii).

First assume that graph $H$ arises from graph $G$ by deleting an edge $e=u w$. We may assume that $G$ has vertex set $V=\{1, \ldots, n\}$, and that $u=1$ and $w=2$. Let $W:=\{3, \ldots, n\}$. Let $f: \mathbb{R} \times \mathcal{O}_{H} \rightarrow \mathcal{S}_{n}$ be defined by

$$
f(h, K):=\left(\begin{array}{ccc}
k_{1,1} & h & K_{\{1\} \times W} \\
h & k_{2,2} & K_{\{2\} \times W} \\
K_{W \times\{1\}} & K_{W \times\{2\}} & K_{W \times W}
\end{array}\right),
$$

where $K=\left(k_{i, j}\right) \in \mathcal{O}_{H}$. Let $f_{0}(K):=f(0, K)$.

Let $M^{\prime}=\left(m_{i, j}^{\prime}\right)$ satisfy (2), with corank $k=\mu(H)$. By (2)(iii),

$$
f_{0} \#_{M^{\prime}} \mathcal{S}_{n, k},
$$

which implies

$$
f \#\left(0, M^{\prime}\right) \mathcal{S}_{n, k} .
$$

Then by (20), there is a neighbourhood $U$ of $\left(0, M^{\prime}\right)$ in $\mathbb{R} \times \mathcal{O}_{H}$ such that for all $x \in U$

$$
f \#{ }_{x} \mathcal{S}_{n, k} \text {. }
$$

Also by (20), $U \cap\left(\{0\} \times f_{0}^{-1}\left(\mathcal{S}_{n, k}\right)\right)$ is a submanifold of $U \cap\left(\{0\} \times \mathcal{O}_{H}\right)$ of codimension $\frac{1}{2} k(k+1)$ (since the codimension of $\mathcal{S}_{n, k}$ in $\mathcal{S}_{n}$ is $\frac{1}{2} k(k+1)$ ). Moreover, $f^{-1}\left(\mathcal{S}_{n, k}\right) \cap U$ is a submanifold of $U$ of codimension $\frac{1}{2} k(k+1)$. Hence there exists a $(h, L) \in U$ with $h<0$ such that $M:=f(h, L) \in \mathcal{S}_{n, k}$. By taking $(h, L)$ close to $\left(0, M^{\prime}\right)$ we may assume that $M$ has exactly one negative eigenvalue. Since $f \#(h, L) \mathcal{S}_{n, k}, M$ fulfils the Strong Arnol'd Hypothesis ((3)). Hence $M$ satisfies (2), and therefore $\mu(G) \geq \mu(H)$.

This theorem implies:

Theorem 4.2 For any subgraph $H$ of any graph $G$ one has

$$
\mu(H) \leq \mu(G) .
$$

Proof By Theorem 4.1 we can assume that $H$ arises from $G=(V, E)$ by deleting an isolated vertex $v$. Let $M^{\prime}$ be a matrix satisfying (2) with respect to $H$, with $\operatorname{corank}\left(M^{\prime}\right)=\mu(H)$, and let $M$ be the $V \times V$ matrix arising from $M^{\prime}$ by adding 0 's, except in position $(v, v)$, where $M_{v, v}=1$. Then trivially corank $(M)=\operatorname{corank}\left(M^{\prime}\right)$ and $M$ satisfies (2) with respect to $G$. This shows (25). 
This implies:

Theorem 4.3 If $G$ has at least one edge, then

$$
\mu(G)=\max _{K} \mu(K),
$$

where $K$ extends over the components of $G$.

Proof By Theorem 4.2 we know that $\geq$ holds in (26). To see equality, let $M$ be a matrix satisfying (2). Since $G$ has at least one edge, we know $\mu(G)>0$ (since trivially $\mu\left(K_{2}\right)=1$ ), and hence $\operatorname{corank}(M)>0$. Then there is exactly one component $L$ of $G$ with $\operatorname{corank}\left(M_{L}\right)>0$. For suppose that there are two such components, $K$ and $L$. Choose nonzero vectors $x \in \operatorname{ker}\left(M_{K}\right)$ and $y \in \operatorname{ker}\left(M_{L}\right)$. Extend $x$ and $y$ by zeros on the positions not in $K$ and $L$, respectively. Then the matrix $X:=x y^{T}+y x^{T}$ is nonzero and symmetric, has zeros in positions corresponding to edges of $G$, and satisfies $M X=0$. This contradicts the Strong Arnol'd Hypothesis.

So corank $(M)=\operatorname{corank}\left(M_{L}\right)$. Suppose now that $M_{L}$ has no negative eigenvalue. Then 0 is the smallest eigenvalue of $M_{L}$, and hence, by the connectivity of $L$ and the Perron-Frobenius theorem, $\operatorname{corank}\left(M_{L}\right)=1$. So $\mu(G)=1$. Let $L^{\prime}$ be a component of $G$ with at least one edge. Then $\mu\left(L^{\prime}\right) \geq 1$, proving (26).

One easily shows that $M_{L}$ satisfies the Strong Arnol'd Hypothesis, implying $\mu(G)=\mu(L)$, thus proving $(26)$

Next we have:

Theorem 4.4 Let $G=(V, E)$ be a graph and let $v \in V$ such that $G-v$ has at least one edge. Then

$$
\mu(G) \leq \mu(G-v)+1
$$

Proof Let $M$ be a matrix satisfying (2) with $\operatorname{corank}(M)=\mu(G)$. Let $M^{\prime}:=$

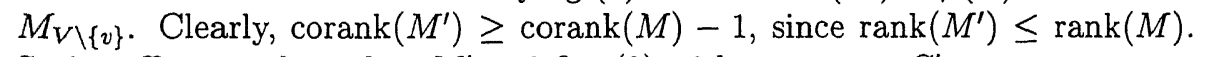
So it suffices to show that $M^{\prime}$ satisfies (2) with respect to $G^{\prime}$.

Trivially, $M^{\prime}$ satisfies (2)(ii). To see that $M^{\prime}$ satisfies (2)(i), it suffices to show that $M^{\prime}$ has at least one negative eigenvalue. If $M^{\prime}$ has no negative eigenvalue, then $M^{\prime}$ is positive semidefinite, and 0 is an eigenvalue of multiplicity at least $\mu(G)+1$. Hence (by the Perron-Frobenius theorem) for each component $K$ of $G-v$, if the matrix $M_{K}$ has eigenvalue 0 , then it has multiplicity 1 . As the theorem trivially holds if $\mu(G) \leq 2$ (since $\mu(G-v) \geq 1$ as $G-v$ has at least one edge), we can assume that $\mu(G) \geq 3$. Hence $G-v$ has at leas $\mu(G)+1 \geq 4$ components $K$ with $M_{K}$ singular. Let $K_{1}, \ldots, K_{4}$ be four suc components. For $i=1, \ldots, 4$, let $x_{i}$ be a nonzero vector with $M_{K_{i}} x_{i}=0$. E the Perron-Frobenius theorem we know that we can assume $x_{i}>0$ for each Extend $x_{i}$ to a vector in $\mathbb{R}^{V}$ by adding components 0 . 
Let $z$ be an eigenvector of $M$ belonging to the smallest eigenvalue of $M$. By scaling the $x_{i}$ we can assume that $z^{T} x_{i}=1$ for each $i$. Now define

$$
X:=\left(x_{1}-x_{2}\right)\left(x_{3}-x_{4}\right)^{T}+\left(x_{3}-x_{4}\right)\left(x_{1}-x_{2}\right)^{T} .
$$

Then $M X=0$, since $M\left(x_{1}-x_{2}\right)=0\left(\right.$ as $\left(x_{1}-x_{2}\right)^{T} M\left(x_{1}-x_{2}\right)=0$ and as $x_{1}-x_{2}$ is orthogonal to $z$ ), and similarly $M\left(x_{3}-x_{4}\right)=0$. This contradicts the fact that $M$ satisfies (2)(iii). So $M^{\prime}$ satisfies (2)(i).

To see that $M^{\prime}$ satisfies the Strong Arnol'd Hypothesis (2)(iii), let $X^{\prime}$ be a $(V \backslash v) \times(V \backslash v)$ matrix with 0 's in positions $(i, j)$ where $i=j$ or $i$ and $j$ are adjacent, and satisfying $M^{\prime} X^{\prime}=0$. We must show that $X^{\prime}=0$. Let $X$ be the $V \times V$ matrix obtained from $X^{\prime}$ by adding 0 's.

Since $M^{\prime}$ has exactly one negative eigenvalue, we know by interlacing that $\operatorname{corank}\left(M^{\prime}\right) \leq \operatorname{corank}(M)$. If $M X=0$ we know by (2)(iii) that $X=0$ and hence $X^{\prime}=0$. So we can assume that $M X \neq 0$. As $\operatorname{corank}\left(M^{\prime}\right) \leq \operatorname{corank}(M)$, it follows that there is a vector $x \in \operatorname{ker}(M)$ with $x_{v} \neq 0$. Hence the first column of $M$ is a linear combination of the other columns of $M$. Therefore $M X=0$, a contradiction.

On the other hand we have, where $S(G)$ arises from $G$ by adding one new vertex $v$ adjacent to all other vertices of $G$ :

Theorem 4.5 For any graph $G$ with at least one edge, one has

$$
\mu(S(G))=\mu(G)+1 .
$$

Proof By Theorem 4.4 it suffices to show that $\mu(S(G)) \geq \mu(G)+1$, and by Theorem 4.3 we can assume that $G$ is connected. Let $M$ be a matrix satisfying (2) with corank $(M)=\mu(G)$. Let $z$ be an eigenvector of $M$ belonging to the smallest eigenvalue $\lambda_{1}$ of $M$. We can assume that $z<0$ and that $\|z\|=1$. Let $M^{\prime}$ be the matrix

$$
M^{\prime}:=\left(\begin{array}{cc}
\lambda_{1}^{-1} & z^{T} \\
z & M
\end{array}\right)
$$

Since $(0, x)^{T} \in \operatorname{ker}\left(M^{\prime}\right)$ for each $x \in \operatorname{ker}(M)$ and since $\left(-\lambda_{1}, z\right)^{T} \in \operatorname{ker}\left(M^{\prime}\right)$, we know that $\operatorname{corank}\left(M^{\prime}\right) \geq \operatorname{corank}(M)+1$. By interlacing it follows that $M^{\prime}$ has exactly one negative eigenvalue. One similarly easily checks that $M^{\prime}$ satisfies the Strong Arnol'd Hypothesis (2)(iii).

Above we gave a proof that $\mu(G)$ is monotone under taking subgraphs. More strongly, as Colin de Verdière [5] proved, $\mu(G)$ is minor-monotone. Again we give the elementary proof due to van der Holst [11] of this fact.

Theorem 4.6 $\mu(G)$ is minor-monotone. 
Proof By Theorem 4.2 it suffices to show that $\mu(H) \leq \mu(G)$ if $H$ arises from $G$ by contracting edge $e=u w$. Let the new vertex of $H$ be $v$. Let $n:=|V|$ and $n^{\prime}:=\left|V^{\prime}\right|$. So $n=n^{\prime}+1$. We may assume that $u=1$ and $w=2$. Let $W:=\{3, \ldots, n\}$.

Let $\mathcal{Z}$ be the set of all matrices $K=\left(k_{i, j}\right) \in \mathcal{O}_{G}$ with $k_{1,1}=0=k_{1,2}$. Define a function

$$
f: \mathbb{R} \times \mathcal{Z} \rightarrow \mathcal{S}_{n^{\prime}}
$$

by

$$
f(h, K)=\left(\begin{array}{cc}
k_{2,2} & K_{\{1\} \times W}+K_{\{2\} \times W} \\
K_{W \times\{1\}}+K_{W \times\{2\}} & K_{W \times W}-h K_{W \times\{1\}} K_{\{1\} \times W}
\end{array}\right),
$$

and let $f_{0}(K)=f(0, K)$.

Let $M^{\prime}=\left(m_{i, j}^{\prime}\right)$ satisfy (2) with respect to $H$, with corank $k=\mu(H)$. Trivially there is a $P \in \mathcal{Z}$ such that $f(0, P)=M^{\prime}$. Since the tangent space of $\mathcal{O}_{H}$ at $M^{\prime}$ is a subspace of the space of all vectors $D f_{(0, P)}(A)$ with $A \in$ $T_{(0, P)}(\mathbb{R} \times \mathcal{Z})$ we know that

$$
f \#(0, P) \mathcal{S}_{n^{\prime}, k} .
$$

Again by (20), there is a neighbourhood $U$ of $(0, P)$ such that for all $x \in U$

$$
f \#{ }_{x} \mathcal{S}_{n^{\prime}, k} .
$$

Also by (20), $\left(\{0\} \times f_{0}^{-1}\left(\mathcal{S}_{n^{\prime}, k}\right)\right) \cap U$ is a submanifold of $U \cap\left(\{0\} \times \mathcal{O}_{H}\right)$ of codimension $\frac{1}{2} k(k+1)$ (since the codimension of $\mathcal{S}_{n^{\prime}, k}$ in $\mathcal{S}_{n^{\prime}}$ is $\frac{1}{2} k(k+1)$ ). Moreover, $f^{-1}\left(\mathcal{S}_{n^{\prime}, k}\right) \cap U$ is a submanifold of $U$ of codimension $\frac{1}{2} k(k+1)$. Hence there is an $(h, L) \in U$ with $h>0$ such that $f(h, L) \in \mathcal{S}_{n^{\prime}, k}$ and

$$
f \#(h, L) \mathcal{S}_{n^{\prime}, k} .
$$

Taking $(h, L)$ close to $(0, P)$ we may assume that $f(h, L)$ has exactly one negative eigenvalue.

Define

$$
M:=\left(\begin{array}{ccc}
\frac{1}{h} & -\frac{1}{h} & L_{\{1\} \times W} \\
-\frac{1}{h} & L_{\{2\} \times\{2\}}+\frac{1}{h} & L_{\{2\} \times W} \\
L_{W \times\{1\}} & L_{W \times\{2\}} & L_{W \times W}
\end{array}\right) .
$$

Clearly $M \in \mathcal{O}_{G}$. We show that $M$ satisfies (2) and has corank $k$. Let

$$
P:=\left(\begin{array}{ccc}
1 & 1 & -h L_{\{1\} \times W} \\
0 & 1 & 0 \\
0 & 0 & I
\end{array}\right)
$$


Then

$$
P^{T} M P=\left(\begin{array}{cc}
\frac{1}{h} & 0 \\
0 & f(h, L)
\end{array}\right)
$$

Therefore, by Sylvester's law of inertia and since $\frac{1}{h}>0, f(h, L)$ has the same number of negative eigenvalues and the same corank as $M$. It remains to show that $M$ fulfils the Strong Arnol'd Hypothesis ((2)(iii)).

Choose $F \in \mathcal{S}_{n}$. We must show that there exists an $N \in T_{M} \mathcal{O}_{G}$ such that $x^{T} F x=x^{T} N x$ for all $x \in \operatorname{ker}(M)$. Define

$$
Q:=\left(\begin{array}{cc}
1 & -h L_{\{1\} \times W} \\
1 & 0 \\
0 & I
\end{array}\right)
$$

and $F^{\prime}:=Q^{T} F Q$.

Since $f \#(h, L) \mathcal{S}_{n^{\prime}, k}$,

$$
D f_{(h, L)}\left(T_{(h, L)}(\mathbb{R} \times \mathcal{Z})\right)+T_{f(h, L)}\left(\mathcal{S}_{n^{\prime}, k}\right)=\mathcal{S}_{n^{\prime}}
$$

The tangent space of $\mathcal{S}_{n^{\prime}, k}$ at $f(h, L)$ is the set of all real-valued symmetric matrices $C$ for which $x^{\prime T} C x^{\prime}=0$ for all $x^{\prime} \in \operatorname{ker}(f(h, L))$. Hence there is an $(a, B) \in T_{(h, L)}(\mathbb{R} \times \mathcal{Z})$ such that

$$
x^{\prime T} D f_{(h, L)}(a, B) x^{\prime}=x^{\prime T} F^{\prime} x^{\prime}
$$

for all $x^{\prime} \in \operatorname{ker}(f(h, L))$.

Now let

$$
N:=\left(\begin{array}{ccc}
\frac{a}{h^{2}} & -\frac{a}{h^{2}} & B_{\{1\} \times W} \\
-\frac{a}{h^{2}} & B_{\{2\} \times\{2\}}+\frac{a}{h^{2}} & B_{\{2\} \times W} \\
B_{W \times\{1\}} & B_{W \times\{2\}} & B_{W \times W}
\end{array}\right) .
$$

So $N \in T_{M} \mathcal{O}_{N}$. A calculation shows

$$
D f_{(h, L)}(a, B)=Q^{T} N Q .
$$

For each vector $x \in \operatorname{ker}(M)$, the vector

$$
x^{\prime}=\left(\begin{array}{c}
x_{2} \\
x_{W}
\end{array}\right)
$$

belongs to $\operatorname{ker}(f(h, L))$ and satisfies $Q x^{\prime}=x$. Hence

$$
x^{T} F x=x^{\prime T} Q^{T} F Q x^{\prime}=x^{\prime T} D f_{(h, L)}(a, B) x^{\prime}=x^{T} N x .
$$




\section{$5 \mu(G)$ and $\lambda(G)$ for complete graphs}

It is easy to see that for each graph $G$ with $n$ vertices one has

$$
\mu(G) \leq n-1 \text { and } \lambda(G) \leq n-1 .
$$

This follows from the fact that any matrix $M$ satisfying (2) has a negative eigenvalue, and that the all-one vector does not belong to any representative subspace $X$ of $\mathbb{R}^{V}$.

Moreover:

Theorem 5.1 For any graph $G$ with $n$ vertices, $\mu(G)=n-1$ if and only if $G$ is complete or $n \leq 2$.

Proof Let $G$ have $n$ vertices. To see sufficiency, first note that trivially $\mu(G)=$ $n-1$ if $n \leq 2$. Moreover $\mu\left(K_{n}\right)=n-1$ follows from the fact that the all -1 matrix satisfies (2) and has corank $n-1$.

To see necessity, let $n \geq 3$ and $\mu(G)=n-1$. Let $M$ be a matrix satisfying (2) with corank $n-1$. So $M$ has rank 1.

Suppose that $M$ has an all-zero row, say row 1 . Then 1 is an isolated vertex of $G$. Since $M$ has rank 1, the dimension of the kernel of $M$ is at least 2, and hence there is a nonzero vector $x \in \mathbb{R}^{n}$ with $x_{1}=0$ and $M x=0$. Let $y \in \mathbb{R}^{n}$ be given by $y_{1}:=1$ and $y_{i}:=0$ for $i>1$. Let $X:=x y^{T}+y x^{T}$. Then $M X=0$, and hence by (2)(iii), $X=0$, a contradiction.

So $M$ does not have any all-zero row, and hence (as $M$ has rank 1), all entries in $M$ are nonzero. So $G$ is complete.

It follows that for each $t \geq 1$, the graph $K_{t+2}$ is a forbidden minor for the property $\mu(G) \leq t$.

Similarly, one has:

Theorem 5.2 For any graph $G$ with $n$ vertices, $\lambda(G)=n-1$ if and only if $G$ is complete or $n \leq 2$.

Proof To see sufficiency, if $n \leq 2$, then trivially $\lambda(G)=n-1$. If $G=K_{n}$, then $\lambda(G) \geq n-1$, since the set $X$ of functions $x \in \mathbb{R}^{V}$ with $\sum_{v \in V} x(v)=0$ is representative for $K_{n}$.

To see necessity, let $n \geq 3$ and $\lambda(G)=n-1$. Suppose that $G$ is not complete, and let vertices $u$ and $u^{\prime}$ be nonadjacent. Let $X$ be a subspace of dimension $n-1$ representative for $G$. So there is a nonzero vector $c \in \mathbb{R}^{V}$ such that $X$ consists of all vectors $x \in \mathbb{R}^{V}$ with $c^{T} x=0$. We can assume that $c_{u}=1$. Then each entry of $c$ is positive. For suppose that $c_{w} \leq 0$. Then the vector $x$ with $x_{w}=1, x_{u}=-c_{w}$, and $x_{v}=0$ for all other vertices $v$, belongs to $X$. So $\operatorname{supp}^{-}(x)=\emptyset$, contradicting (10).

Now by scaling we can assume that each entry in $c$ is 1 . Let $v$ be any vertex different from $u$ and $u^{\prime}$. Then for the vector $x$ with $x_{u}=1, x_{u^{\prime}}=1, x_{w}=-2$, and $x_{v}=0$ for all other vertices $v$, the graph $G \mid \operatorname{supp}^{+}(x)$ is disconnected, contradicting (10). 
Hence, for each $t \geq 1$, the graph $K_{t+2}$ is a forbidden minor for the property $\lambda(G) \leq t$.

\section{Clique sums}

As mentioned, Colin de Verdière conjectures that $\gamma(G) \leq \mu(G)+1$, where $\gamma(G)$ is the colouring number of $G$. This conjecture would follow from Hadwiger's conjecture (as $\mu\left(K_{n}\right)=n-1$ ), and is true for $\mu(G) \leq 4$. A similar relation holds for the size of the largest clique minor in a graph. We therefore are interested in studying the behaviour of $\mu(G)$ and $\lambda(G)$ under clique sums.

To study this for $\lambda(G)$, we first give an auxiliary result. For any finite subset $Z$ of $\mathbb{R}^{d}$ let cone $(Z)$ denote the smallest nonempty convex cone containing $Z$; that is, it is the intersection of all closed halfspaces $\left\{x \in \mathbb{R}^{d} \mid c^{T} x \geq 0\right\}$ containing $Z$. (Thus cone $(\emptyset)=\{0\}$, while $\operatorname{cone}(Z)=\mathbb{R}^{d}$ if there are no halfspaces containing $Z$.)

For any graph $G=(V, E)$ and $U \subseteq V$, let $G-U$ denote the graph obtained from $G$ by deleting the vertices of $U$. (So $G-U=G \mid(V \backslash U)$.)

Theorem 6.1 Let $\phi: V \rightarrow \mathbb{R}^{d}$ be representative for a graph $G=(V, E)$ and let $U \subseteq V$. Assume that cone $(\phi(U))$ is not a hyperplane in $\mathbb{R}^{d}$. Then there is at most one component $K$ of $G-U$ for which the inclusion $\phi(K) \subseteq \operatorname{cone}(\phi(U))$ does not hold.

Proof We may assume that cone $(\phi(U)) \neq \mathbb{R}^{d}$. Since cone $(\phi(U))$ is not a hyperplane in $\mathbb{R}^{d}$, the set

$$
C:=\left\{c \in \mathbb{R}^{d} \mid c \neq 0, c^{T} \phi(v) \leq 0 \text { for each } v \in U\right\},
$$

is nonempty and topologically connected (because the polar cone $C \cup\{0\}$ of cone $(\phi(U))$ is not a line). For $c \in \mathbb{R}^{d}$, let $H_{c}:=\left\{x \in \mathbb{R}^{d} \mid c^{T} x>0\right\}$. Let $K_{1}, \ldots, K_{t}$ be the components of $G-U$. Let $C_{i}$ be the set of vectors $c \in C$ for which $H_{c}$ intersects $\phi\left(K_{i}\right)$. So if $i \neq j$ then $C_{i} \cap C_{j}=\emptyset$, since if $c \in C$ then $\phi^{-1}\left(H_{c}\right)$ is connected and is disjoint from $U$. As $C_{1} \cup \cdots \cup C_{t}=C$ and since each $C_{i}$ is an open subset of $C$, it follows that $C_{i}=\emptyset$ for all but one $i$. Hence $\phi\left(K_{i}\right) \subseteq \operatorname{cone}(\phi(U))$ for all but one $i$.

This implies ([12]):

Theorem 6.2 If $G$ has at least one edge and is a clique sum of $G_{1}$ and $G_{2}$, then

$$
\lambda(G)=\max \left\{\lambda\left(G_{1}\right), \lambda\left(G_{2}\right)\right\} .
$$

Proof We have $\lambda(G) \geq \max \left\{\lambda\left(G_{1}\right), \lambda\left(G_{2}\right)\right\}$, since $G_{1}$ and $G_{2}$ are subgraphs of $G$, So it suffices to show that $\lambda(G)=\lambda\left(G_{i}\right)$ for some $i=1,2$. Assume that $\lambda(G)>\max \left\{\lambda\left(G_{1}\right), \lambda\left(G_{2}\right)\right\}$. Let $d:=\lambda(G), G=(V, E)$, and $G_{i}=\left(V_{i}, E_{i}\right)$ for $i=1,2$. 
Let $\phi: V \rightarrow \mathbb{R}^{d}$ be representative for $G$. As $d>\lambda\left(G_{i}\right), \phi \mid V_{i}$ is not representative for $G_{i}$, for $i=1$ and $i=2$. Let $K:=V_{1} \cap V_{2}$ and $t:=|K|$. We may assume that we have chosen the counterexample so that $|K|$ is as small as possible.

Then $G \mid\left(V_{1} \backslash K\right)$ has a component $L$ such that each vertex in $K$ is adjacent to at least one vertex in $L$. Otherwise $G$ would be a repeated clique sum of subgraphs of $G_{1}$ and $G_{2}$ with common clique being smaller than $K$. In that case $\lambda(G)=\max \left\{\lambda\left(G_{1}\right), \lambda\left(G_{2}\right)\right\}$ would follow by the minimality of $K$.

So $G_{1}$ has a $K_{t+1}$-minor. So $\lambda\left(G_{1}\right) \geq t$, and hence $\lambda(G)>t=|K|$. Therefore, cone $(\phi(K))$ is not a hyperplane in $\mathbb{R}^{d}$. (Here we use that it is not the case that $K=\emptyset$ and $d=1$.) So by Theorem 6.1, we may assume that $\phi\left(V_{1}\right) \subseteq \operatorname{cone}(\phi(K))$.

As $d>\lambda\left(G_{2}\right)$, there exists a halfspace $H$ of $\mathbb{R}^{d}$ such that $G \mid\left(\phi^{-1}(H) \cap V_{2}\right)$ is empty or disconnected. If it is empty, then $\phi(v) \in H$ for some $v \in V_{1} \backslash K$, contradicting the facts that $\phi(v) \in \operatorname{cone}(\phi(K))$ and that $\phi(K) \cap H=\emptyset$. So it is disconnected. But then also $\phi^{-1}(H)$ would induce a disconnected subgraph of $G$, as $K$ is a clique. This is a contradiction.

Hence we have that for each $t \geq 1$ :

the class of graphs $G$ with $\lambda(G) \leq t$ is closed under taking clique sums.

A statement like this for $\mu$ does not hold. A critical example is the graph $K_{t+3} \backslash \Delta$ (the graph obtained from the complete graph $K_{t+3}$ by deleting the edges of a triangle). One has $\mu\left(K_{t+3} \backslash \Delta\right)=t+1$ (since the star $K_{4} \backslash \Delta$ has $\mu\left(K_{4} \backslash \Delta\right)=2$ (see Theorem 8.2 below), and since adding a new vertex adjacent to all existing vertices increases $\mu$ by 1).

However, $K_{t+3} \backslash \Delta$ is a clique sum of $K_{t+1}$ and $K_{t+2} \backslash e$ (the graph obtained from $K_{t+2}$ by deleting an edge), with common clique of size $t$. Both $K_{t+1}$ and $K_{t+2} \backslash e$ have $\mu=t$. So, generally one does not have that, for fixed $t$, the property $\mu(G) \leq t$ is maintained under clique sums. Similarly, $K_{t+3} \backslash \Delta$ is a clique sum of two copies of $K_{t+2} \backslash e$, with common clique of size $t+1$.

These examples where $\mu$ increases by taking a clique sum are in a sense the only cases, as shown in [13]:

Theorem 6.3 If $G$ has at least one edge and is a clique sum of $G_{1}$ and $G_{2}$, with common clique $S$, then $\mu(G)>t:=\max \left\{\mu\left(G_{1}\right), \mu\left(G_{2}\right)\right\}$ if and only if:

either (i) $|S|=t$ and $G-S$ has three components the contraction of which makes with $S$ a $K_{t+3} \backslash \Delta$,

or (ii) $|S|=t+1$ and $G-S$ has two components the contraction of which makes with $S$ a $K_{t+3} \backslash \Delta$.

Moreover, if $\mu(G)>t$ then $\mu(G)=t+1, \mu\left(G_{1}\right)=\mu\left(G_{2}\right)=t$, and we can contract two or three components of $G-S$ so that the contracted vertices together with $S$ form a $K_{t+3} \backslash \Delta$. 


\section{Behaviour of $\mu(G)$ and $\lambda(G)$ under $\mathrm{Y} \Delta$ and $\Delta \mathrm{Y}$}

The results on clique sums can be applied to study the behaviour of $\mu(G)$ and $\lambda(G)$ under applying the $\mathrm{Y} \Delta$ - and $\Delta \mathrm{Y}$-operations. The $\mathrm{Y} \Delta$-operation works as follows, on a graph $G$ : choose a vertex $v$ of degree 3 , make its three neighbours pairwise adjacent, and delete $v$ and the three edges incident with $v$. The $\Delta Y$-operation is the reverse operation, starting with a triangle and adding a new vertex.

Note that the if $H$ arises by a $\Delta \mathrm{Y}$ from $G$, then $H$ is a subgraph of a clique sum of $G$ and $K_{4}$. Then Theorem 6.3 implies that $\mu(H) \leq \mu(G)$ if $\mu(G) \geq 4$, and Theorem 6.2 that $\lambda(H) \leq \lambda(G)$ if $\lambda(G) \geq 3$.

In fact, Bacher and Colin de Verdière [1] proved:

Let $H$ arise by a $\Delta \mathrm{Y}$ operation from $G$. Then $\mu(H) \leq \mu(G)$.

If moreover $\mu(G) \geq 4$, then $\mu(H)=\mu(G)$.

\section{$8 \mu(G)$ and $\lambda(G)$ for complete bipartite graphs}

Since complete bipartite graphs are often candidates for forbidden minors, in this section we give formulas for $\mu\left(K_{m, n}\right)$ and $\lambda\left(K_{m, n}\right)$. This also exhibits a difference between $\mu(G)$ and $\lambda(G)$. First we consider $\lambda(G)$ :

Theorem 8.1 For $n \geq m \geq 1, \lambda\left(K_{m, n}\right)=m$.

Proof On the one hand, $K_{m+1}$ is a minor of $K_{m, n}$, and on the other hand, $K_{m, n}$ is a subgraph of a clique sum of $K_{m+1}$ 's. So by Theorem $6.2 \lambda\left(K_{m, n}\right)=$ $\lambda\left(K_{m+1}\right)=m$.

Characterizing $\mu(G)$ for complete bipartite graphs is a little more complicated:

Theorem 8.2 For $n \geq m \geq 1$ we have

$$
\mu\left(K_{m, n}\right)= \begin{cases}m & \text { if } n \leq 2 \\ m+1 & \text { if } n \geq 3\end{cases}
$$

Proof Note that $\mu\left(K_{m, n}\right) \leq m+1$ by Theorem 6.3 , since $K_{m, n}$ is a subgraph of a clique sum of $K_{m+1}$ 's. It is not hard to see that $\mu\left(K_{1,1}\right)=\mu\left(K_{1,2}\right)=1$ and $\mu\left(K_{2,2}\right)=2$. Hence $\mu\left(K_{m, n}\right)=m$ if $n \leq 2$.

So let $n \geq 3$. If $m \leq 3$ we can assume that $n=3$. Let $K_{m, 3}$ have vertices $1, \ldots, m+3$, with colour classes $\{1, \ldots, m\}$ and $\{m+1, m+2, m+3\}$. Let $M$ be the $(m+3) \times(m+3)$ matrix with $m_{i, j}=-1$ if $i \leq m<j$ or $j \leq m<i$, and $m_{i, j}=0$ otherwise. Then $M$ has rank 2 and hence corank $m+1$. Moreover $M$ satisfies (2). Indeed, (2)(ii) is trivial. Moreover, (2)(i) follows directly from the fact that neither $M$ nor $-M$ is positive semi-definite. Finally, 
$M$ satisfies the Strong Arnol'd Hypothesis ((2)(iii)). Otherwise there is a nonzero symmetric matrix $X$ with $M X=0$ and $x_{i, j}=0$ if $i=j$ or $i \leq m<j$, which can be seen to be impossible using the fact that $m \leq n=3$.

If $m \geq 4$, we can assume that $n=m$. Choose two adjacent vertices $u$ and $v$ of $K_{m, m}$. Delete the edge $u v$, and delete $m-4$ other edges incident with $u$ and $m-4$ other edges incident with $v$. So in the new graph, $u$ and $v$ have degree 3 . Applying Y $\Delta$ to $u$ and to $v$ we obtain a $K_{m-1, m-1}$ with a triangle added to each of the colour classes. The $2(m-4)$ vertices not covered by these triangles span a matching of size $m-4$. Contracting each edge of this matching, we obtain a $K_{m+2}$. Since $\mu\left(K_{m+2}\right)=m+1$, we obtain $\mu\left(K_{m, m}\right)=m+1$ (using (51)).

\section{Characterizing $\lambda(G) \leq 1$ and $\mu(G) \leq 1$}

Note that one trivially has:

$$
\mu(G)=0 \quad \Longleftrightarrow \quad \lambda(G)=0 \quad \Longleftrightarrow \quad G \text { has exactly one vertex. }
$$

We next describe the collections of graphs $G$ satisfying $\mu(G) \leq 1$ and $\lambda(G) \leq 1$. For $\mu(G)$ it is $([5])$ :

Theorem $9.1 \mu(G) \leq 1$ if and only if $G$ is a vertex-disjoint union of paths; that is, if $G$ does not have a $K_{3}$ or $K_{1,3}$-minor.

Proof Since $\mu\left(K_{3}\right)=2$ by Theorem 5.1 and $\mu\left(K_{1,3}\right)=2$ by Theorem 8.2, the minor-monotonicity of $\mu$ gives the 'only if' part.

To see the 'if' part, we can assume, by the minor-monotonicity of $\mu(G)$, that $G$ is a path. Then trivially any matrix $M$ satisfying (2) has rank at least $n-1$, and hence corank at most 1 . So $\mu(G) \leq 1$.

The class of graphs $G$ with $\lambda(G) \leq 1$ is a little larger ([12]):

Theorem $9.2 \lambda(G) \leq 1$ if and only if $G$ is a forest; that is, if and only if $G$ does not have a $K_{3}$-minor.

Proof If $\lambda(G) \leq 1$ then $G$ has no $K_{3}$-minor, as $\lambda\left(K_{3}\right)=2$. Conversely, if $G$ is a forest, then $G$ arises by taking clique sums and subgraphs from the graph $K_{2}$. As $\lambda\left(K_{2}\right)=1$, Theorem 6.2 gives the corollary.

\section{Van der Holst's lemma}

In characterizing $\mu(G) \leq 2$ and $\mu(G) \leq 3$ the lemma due to van der Holst [10] turns out to be very helpful.

If $x \in \mathbb{R}^{n}$ and $I \subseteq\{1, \ldots, n\}$, then $x_{I}$ denotes the subvector of $x$ induced by the indices in $I$.

Recall that a vector $x \in \operatorname{ker}(M)$ has minimal support if $x$ is nonzero and for each nonzero vector $y \in \operatorname{ker}(M)$ with $\operatorname{supp}(y) \subseteq \operatorname{supp}(x)$ one has $\operatorname{supp}(y)=$ $\operatorname{supp}(x)$. 
Theorem 10.1 (Van der Holst's lemma) Let $G$ be a connected graph and let $M$ satisfy (2). Let $x \in \operatorname{ker}(M)$ have minimal support. Then $G \mid \operatorname{supp}^{+}(x)$ and $G \mid \operatorname{supp}^{-}(x)$ are both connected.

Proof Suppose that (say) $G \mid \operatorname{supp}^{+}(x)$ is disconnected. Let $I$ and $J$ be two of the components of $G \mid \operatorname{supp}^{+}(x)$. Let $K:=\operatorname{supp}^{-}(x)$. Since $m_{i, j}=0$ if $i \in I$, $j \in J$, we have:

$$
\begin{aligned}
M_{I \times I} x_{I}+M_{I \times K} x_{K} & =0, \\
M_{J \times J} x_{J}+M_{J \times K} x_{K} & =0 .
\end{aligned}
$$

Let $z$ be an eigenvector of $M$ with negative eigenvalue. By the PerronFrobenius theorem we may assume $z>0$. (Strictly speaking, we apply the Perron-Frobenius theorem to the (nonnegative and indecomposable) matrix $\lambda I-M$ choosing $\lambda$ large enough.)

Let

$$
\lambda:=\frac{z_{I}^{T} x_{I}}{z_{J}^{T} x_{J}} .
$$

Define $y \in \mathbb{R}^{n}$ by: $y_{i}:=x_{i}$ if $i \in I, y_{i}:=-\lambda x_{i}$ if $i \in J$, and $x_{i}:=0$ if $i \notin I \cup J$. By (55), $z^{T} y=z_{I}^{T} x_{I}-\lambda z_{J}^{T} x_{J}=0$. Moreover, one has (since $m_{i, j}=0$ if $i \in I$ and $j \in J)$ :

$$
\begin{aligned}
y^{T} M y & =y_{I}^{T} M_{I \times I} y_{I}+y_{J}^{T} M_{J \times J} y_{J} \\
& =x_{I}^{T} M_{I \times I} x_{I}+\lambda^{2} x_{J}^{T} M_{J \times J} x_{J} \\
& =-x_{I}^{T} M_{I \times K} x_{K}-\lambda^{2} x_{J}^{T} M_{J \times K} x_{K} \\
& \leq 0,
\end{aligned}
$$

(using (54)) since $M_{I \times K}$ and $M_{J \times K}$ are nonpositive, and since $x_{I}>0, x_{J}>0$ and $x_{K}<0$.

Now $z^{T} y=0$ and $y^{T} M y \leq 0$ imply that $M y=0$ (as $M$ is symmetric and has exactly one negative eigenvalue, with eigenvector $z$ ). Therefore, $y \in$ $\operatorname{ker}(M)$. This contradicts the fact that $x$ has minimal support.

We note that if $M$ satisfies (2), then each vertex $v \notin \operatorname{supp}(x)$ adjacent to some vertex in $\operatorname{supp}^{+}(x)$ is also adjacent to some vertex in $\operatorname{supp}^{-}(x)$, and conversely; that is,

$$
\begin{aligned}
& \text { for each } x \in \operatorname{ker}(M) \text { : } \\
& N\left(\operatorname{supp}^{+}(x)\right) \backslash \operatorname{supp}(x)=N\left(\operatorname{supp}^{-}(x)\right) \backslash \operatorname{supp}(x) .
\end{aligned}
$$

Here $N(U)$ is the set of vertices in $V \backslash U$ that are adjacent to at least one vertex in $U$. 


\section{Characterizing $\mu(G) \leq 2$ and $\lambda(G) \leq 2$}

We can now derive the following result of Colin de Verdière [5]:

Theorem $11.1 \mu(G) \leq 2$ if and only if $G$ is outerplanar; that is, if and only if $G$ does not have a $K_{4}$ - or $K_{2,3}$-minor.

Proof Since $\mu\left(K_{4}\right)=3$ by Theorem 5.1 and $\mu\left(K_{2,3}\right)=3$ by Theorem 8.2 , the minor-monotonicity of $\mu$ gives the 'only if' part (using the forbidden minor characterization of outerplanarity).

To see the 'if' part, we may assume that $G$ is maximally outerplanar. Suppose that $\mu(G)>2$, and let $M$ be a matrix satisfying (2) of corank more than 2. Let $u v$ be a boundary edge of $G$. Then there exists a nonzero vector $x \in \operatorname{ker}(M)$ with $x_{u}=x_{v}=0$. We can assume that $x$ has minimal support. By Van der Holst's lemma (Theorem 10.1), $G \mid \operatorname{supp}^{+}(x)$ and $G \mid \operatorname{supp}^{-}(x)$ are nonempty and connected. As $G$ is maximally outerplanar, $G$ is 2-connected. Hence there exist two vertex-disjoint paths $P_{1}$ and $P_{2}$ from $\operatorname{supp}(x)$ to $\{u, v\}$. Let $P_{1}^{\prime}$ and $P_{2}^{\prime}$ be the parts outside $\operatorname{supp}(x)$. Then the first vertices of $P_{1}^{\prime}$ and $P_{2}^{\prime}$ both belong to $N(\operatorname{supp}(x))$, and hence (by (57)) to both $N\left(\operatorname{supp}^{+}(x)\right)$ and $N\left(\operatorname{supp}^{-}(x)\right)$. Contracting each of supp ${ }^{+}(x), \operatorname{supp}^{-}(x), P_{1}^{\prime}$, and $P_{2}^{\prime}$ to one point, gives an embedded outerplanar graph with $u v$ on the boundary and $u$ and $v$ connected by two paths of length two. This is not possible. $[12]:$

The corresponding characterization for $\lambda(G)$ is easier, and was given in

Theorem $11.2 \lambda(G) \leq 2$ if and only if $G$ is a series-parallel graph; that is, if and only if $G$ does not have a $K_{4}$-minor.

Proof If $\lambda(G) \leq 2$ then $G$ has no $K_{4}$-minor, as $\lambda\left(K_{4}\right)=3$.

Conversely, if $G$ is a series-parallel graph, then $G$ arises by taking clique sums and subgraphs from the graph $K_{3}$. As $\lambda\left(K_{3}\right)=2$, Theorem 6.2 gives the corollary.

\section{Characterizing $\mu(G) \leq 3$}

We apply Van der Holst's lemma (Theorem 10.1) similarly to the case $\mu \leq 3$, a main result of Colin de Verdière [5]:

Theorem 12.1 $\mu(G) \leq 3$ if and only if $G$ is planar; that is, if and only if $G$ does not have a $K_{5}$ - or $K_{3,3}$-minor.

Proof Since $\mu\left(K_{5}\right)=4$ by Theorem 5.1 and $\mu\left(K_{3,3}\right)=4$ by Theorem 8.2 , the minor-monotonicity of $\mu$ gives the 'only if' part (using Kuratowski's forbidden minor characterization of planarity). 
To see the 'if' part, we may assume that $G$ is maximally planar (triangulated). Suppose that $\mu(G)>3$ and let $M$ be a matrix satisfying (2) of corank more than 3. Let $u v w$ be a face of $G$. Then there exists a nonzero vector $x \in \operatorname{ker}(M)$ with $x_{u}=x_{v}=x_{w}=0$. We can assume that $x$ has min. imal support. By Van der Holst's lemma (Theorem 10.1), $G \mid \operatorname{supp}^{+}(x)$ and $G \mid \operatorname{supp}^{-}(x)$ are nonempty and connected. As $G$ is maximally planar, $G$ is 3 -connected. Hence there exist three vertex-disjoint paths $P_{1}, P_{2}, P_{3}$ from $\operatorname{supp}(x)$ to $\{u, v, w\}$. Let $P_{1}^{\prime}, P_{2}^{\prime}, P_{3}^{\prime}$ be the parts outside $\operatorname{supp}(x)$. Then the first vertices of the $P_{i}^{\prime}$ belong to $N(\operatorname{supp}(x))$, and hence (by (57)) to both $N\left(\operatorname{supp}^{+}(x)\right)$ and $N\left(\operatorname{supp}^{-}(x)\right)$. Contracting each of supp ${ }^{+}(x), \operatorname{supp}^{-}(x), P_{1}^{\prime}$, $P_{2}^{\prime}, P_{3}^{\prime}$ to one point, would give an embedded outerplanar graph with uvw forming a face and $u, v$, and $w$ having two common neighbours. This is not possible.

\section{Characterizing $\lambda(G) \leq 3$}

We characterize in this section the graphs $G$ satisfying $\lambda(G) \leq 3$, a result of [12]. The main ingredient is:

Theorem 13.1 If $G$ is planar then $\lambda(G) \leq 3$.

Proof Suppose $G=(V, E)$ is a planar graph with $\lambda(G) \geq 4$ and $|V|$ minimal. We assume that we have an embedding of $G$ in the sphere. For each face $f$ of $G$ let $V_{f}$ be the set of vertices incident with $f$. Note that $G$ is 4-connected. since otherwise it would be a subgraph of clique sums of smaller planar graphs, and hence we would have $\lambda(G) \leq 3$ by Theorem 6.2 .

Let $\phi: V \rightarrow \mathbb{R}^{4}$ be representative for $G$. Then $\phi(v) \neq 0$ for each $v \in V$, since otherwise we can delete $v$, contradicting the minimality of $G$. So we can assume that $\|\phi(v)\|=1$ for each $v \in V$.

We may assume that, for each edge $u v, \phi(u) \neq \pm \phi(v)$, since otherwise, either $\phi(u)=\phi(v)$, in which case we can contract the edge $\{u, v\}$ in $G$, or $\phi(u)=-\phi(v)$, in which case we can delete the edge $\{u, v\}$ from $G$. In either case we obtain a contradiction with the minimality of $G$.

Observe that if $f$ and $f^{\prime}$ are faces with $\operatorname{dim}\left(\phi\left(V_{f}\right)\right)=\operatorname{dim}\left(\phi\left(V_{f^{\prime}}\right)\right)=2$ and having a common edge, $e$ say, then $\operatorname{lin} \cdot \operatorname{hull}\left(\phi\left(V_{f}\right)\right)=\operatorname{lin} \cdot \operatorname{hull}\left(\phi\left(V_{f^{\prime}}\right)\right)$, as it is equal to $\operatorname{lin} \cdot \operatorname{hull}(\phi(e))$. Similarly, $\operatorname{lin} \cdot \operatorname{hull}\left(\phi\left(V_{f}\right)\right) \subseteq \operatorname{lin} \cdot \operatorname{hull}\left(\phi\left(V_{f^{\prime}}\right)\right)$ if $\operatorname{dim}\left(\phi\left(V_{f}\right)\right)=2, \operatorname{dim}\left(\phi\left(V_{f^{\prime}}\right)\right)=3$ and $f, f^{\prime}$ share a common edge.

Fixing $V$, we choose $E$ maximal under the condition that $\phi(u) \neq \pm \phi(v)$ for each edge $\{u, v\}$. Then $\operatorname{dim}\left(\phi\left(V_{f}\right)\right) \in\{2,3\}$ for each face $f$. Indeed, $\operatorname{dim}\left(\phi\left(V_{f}\right)\right) \geq 2$, as each edge $e=u v$ has $\operatorname{dim}(\phi(\{u, v\})) \geq 2$. Moreover, if $\operatorname{dim}\left(\phi\left(V_{f}\right)\right)=4$, then $V_{f}$ contains at least two nonadjacent vertices $u, v$ with $\operatorname{dim}(\phi(\{u, v\}))=2$. As we can add the edge $u v$, this contradicts the maximality of $E$. 
For $c \in \mathbb{R}^{4}$ let

$$
\begin{aligned}
& c^{+}:=\left\{v \in V \mid c^{T} \phi(v)>0\right\}, \\
& c^{-}:=\left\{v \in V \mid c^{T} \phi(v)<0\right\},
\end{aligned}
$$

and let $\mathcal{F}_{c}$ be the set of faces $f$ for which $V_{f}$ intersects both $c^{+}$and $c^{-}$. Then:

Let $f$ and $f^{\prime}$ be two faces with $\operatorname{dim}\left(\phi\left(V_{f} \cup V_{f^{\prime}}\right)\right)=4$. Then there is a $c \in \mathbb{R}^{4}$ with $f, f^{\prime} \in \mathcal{F}_{c}$.

To see this, we note that because $\operatorname{dim}\left(\phi\left(V_{f}\right)\right) \geq 2, \operatorname{dim}\left(\phi\left(V_{f^{\prime}}\right)\right) \geq 2$, and $\operatorname{dim}\left(\phi\left(V_{f} \cup V_{f^{\prime}}\right)\right)=4$, there exist vertices $u, v \in V_{f}$ and $u^{\prime}, v^{\prime} \in V_{f^{\prime}}$ with $\operatorname{dim}\left(\phi\left(\left\{u, v, u^{\prime}, v^{\prime}\right\}\right)\right)=4$. Therefore, we can find a $c \in \mathbb{R}^{4}$ such that $u, u^{\prime} \in c^{+}$ and $v, v^{\prime} \in c^{-}$. So $f, f^{\prime} \in \mathcal{F}_{c}$, proving (59).

For $c \in \mathbb{R}^{4}$, let $W_{c}:=\bigcup\left\{V_{f} \mid f \in \mathcal{F}_{c}\right\}$. To finish the proof of the theorem, it suffices to show:

$$
\operatorname{dim}\left(\phi\left(W_{c}\right)\right) \leq 3 \text { for each } c \in \mathbb{R}^{4} .
$$

This is sufficient, since (60) implies an immediate contradiction with (59), as there exist faces $f$ and $f^{\prime}$ with $\operatorname{dim}\left(V_{f} \cup V_{f^{\prime}}\right)=4$, since $\operatorname{dim}(\phi(V))=4$ and as there is a face $f$ with $\operatorname{dim}\left(\phi\left(V_{f}\right)\right)=3$ (since if $\operatorname{dim}\left(\phi\left(V_{f}\right)\right)=2$ for each face $f$ then $\operatorname{dim}(\phi(V))=2$, since $\operatorname{lin} \cdot h u l l\left(\phi\left(V_{f}\right)\right)=\operatorname{lin} \cdot h u l l\left(\phi\left(V_{f^{\prime}}\right)\right)$ for any two adjacent faces $\left.f, f^{\prime}\right)$.

We show that (60) holds. It suffices to show the result for those $c$ with $W_{c}$ inclusionwise maximal, and hence with $c^{T} \phi(v) \neq 0$ for each vertex $v$.

Let such a $c$ be given. As both $G \mid c^{+}$and $G \mid c^{-}$are connected, the cut $\delta\left(c^{+}\right)$ corresponds in the dual graph of $G$ to a circuit $C$ which traverses exactly two edges in each face $f \in \mathcal{F}_{c}$.

Suppose, to obtain a contradiction, that $\operatorname{dim}\left(\phi\left(W_{c}\right)\right)=4$. Then there exist faces $f, f^{\prime} \in \mathcal{F}_{c}$ with $\operatorname{dim}\left(\phi\left(V_{f}\right)\right)=\operatorname{dim}\left(\phi\left(V_{f^{\prime}}\right)\right)=3$ and such that $\operatorname{lin} \cdot \operatorname{hull}\left(\phi\left(V_{f}\right)\right) \neq \operatorname{lin} \cdot \operatorname{hull}\left(\phi\left(V_{f^{\prime}}\right)\right)$ (as otherwise lin.hull $\left(\phi\left(V_{f}\right)\right)=\operatorname{lin} \cdot \operatorname{hull}\left(\phi\left(V_{f^{\prime}}\right)\right)$ for all $f, f^{\prime} \in \mathcal{F}_{c}$ with $\operatorname{dim}\left(\phi\left(V_{f}\right)\right)=3$ and $\operatorname{dim}\left(\phi\left(V_{f^{\prime}}\right)\right)=3$, which implies that $\left.\operatorname{dim}\left(\phi\left(W_{c}\right)\right)=3\right)$. They correspond to two vertices on $C$. Denote by $f_{1}, \ldots, f_{t}$ the faces between $f$ and $f^{\prime}$ when travelling from $f$ to $f^{\prime}$ along $C$ (in a given direction). Set $f_{0}:=f$ and $f_{t}:=f^{\prime}$. Then we may assume that $\operatorname{dim}\left(\phi\left(V_{f_{i}}\right)\right)=2$ for all $i=1, \ldots, t$. (Otherwise we can make $t$ smaller.)

For $i=0,1, \ldots, t$, let $u_{i} v_{i}$ be the edge common to the faces $f_{i}$ and $f_{i+1}$. So each $u_{i} v_{i}$ belongs to $\delta\left(c^{+}\right)$(as $G$ is 4-connected). We may assume that $u_{i} \in c^{+}$ and $v_{i} \in c^{-}$for each $i$.

Now choose $w \in V_{f}$ so that $\phi(w) \notin \operatorname{lin} \cdot \operatorname{hull}\left(\phi\left(V_{f^{\prime}}\right)\right)$ and $w^{\prime} \in V_{f^{\prime}}$ so that $\phi\left(w^{\prime}\right) \notin \operatorname{lin}$.hull $\left(\phi\left(V_{f}\right)\right)$. Then the set $\phi\left(\left\{u_{0}, v_{0}, w, w^{\prime}\right\}\right)$ has dimension 4 . Hence, there exists a $d \in \mathbb{R}^{4}$ such that $d^{T} \phi(w)>0, d^{T} \phi\left(w^{\prime}\right)>0, d^{T} \phi\left(u_{0}\right)=0$, and $d^{T} \phi\left(v_{0}\right)=0$. Then the set $d^{+} \cup d^{-}$contains none of the vertices on the faces $f_{1}, \ldots, f_{t}$ (since $V_{f_{i}} \subseteq \operatorname{lin}$.hull $\left(\phi\left(\left\{u_{0}, v_{0}\right\}\right)\right)$ for all $\left.i=1, \ldots, t\right)$. In particular, $u_{i}, v_{i} \notin d^{+} \cup d^{-}$for $i=1, \ldots, t$. By the connectivity of $G \mid d^{+}$there exists a path $P$ from $w$ to $w^{\prime}$ which is entirely contained in $d^{+}$. 
Consider the region $R:=\bigcup_{i=0}^{t+1} \bar{f}_{i}$ (where $\bar{f}_{i}$ is the topological closure of $f_{i}$ ). As $P$ joins two vertices on the boundary of $R, R \cup P$ partitions the rest of the sphere into two regions $R_{1}$ and $R_{2}$. We choose indices such that $R_{1}$ has the vertices $u_{0}, \ldots, u_{t}$ on its boundary, while $R_{2}$ has the vertices $v_{0}, \ldots, v_{t}$ on its boundary.

By the connectivity of $G \mid d^{-}, d^{-}$is contained either in $\bar{R}_{1}$ or in $\bar{R}_{2}$. Suppose first that $d^{-}$is contained in $\bar{R}_{1}$. Consider the vector $\tilde{d}=d+\varepsilon c$, with $\varepsilon>0$ small enough such that $d^{+} \subseteq \tilde{d}^{+}$and $d^{-} \subseteq \tilde{d}^{-}$. Then, $\tilde{d}^{-} \supseteq\left\{v_{0}, \ldots, v_{t}\right\} \cup d^{-}$, while $u_{0}, \ldots, u_{t} \in \tilde{d}^{+}$. Then there is no path joining $v_{0}$ and $d^{-}$which is entirely contained in $\tilde{d}^{-}$, contradicting the connectivity of $G \mid \tilde{d}^{-}$.

If $d^{-}$is contained in $\bar{R}_{2}$, we arrive similarly at a contradiction, by considering $\tilde{d}=d-\varepsilon c$.

We can now characterize the graphs $G$ satisfying $\lambda(G) \leq 3$. Having Theorem 13.1, Theorem 6.2 gives that $\lambda(G) \leq 3$ also holds for graphs $G$ obtained from planar graphs by taking clique sums and subgraphs. This characterizes the graphs $G$ with $\lambda(G) \leq 3$, as follows from the following two theorems.

Theorem 13.2 If $G$ has no $K_{5}$ - or $V_{8}$-minor, then $G$ can be obtained by taking clique sums and subgraphs from planar graphs.

Proof Suppose $G$ is not planar. If $G$ is not 3-connected, then it is easy to see that $G$ is a subgraph of a clique sum of two smaller graphs not having a $K_{5}$ or $V_{8}$-minor. So we may assume that $G$ is 3-connected.

Then by Wagner's theorem [20], $G$ can be obtained as a subgraph of a 3 -clique sum of two smaller graphs $G_{1}$ and $G_{2}$ both with no $K_{5}$-minor. Let $K$ be the clique.

It suffices to show that $G_{1}$ and $G_{2}$ have no $V_{8}$-minor. Suppose to the contrary that $G_{1}$, say, has a $V_{8}$-minor. As $V_{8}$ does not contain any triangle, the $V_{8}$-minor in $G_{1}$ does not need all three edges of $K$. So $G_{1}-e$ has a $V_{8}$-minor for some edge $e$ in $K$. However, $G_{1}-e$ is a minor of $G$ (by the 3-connectedness of $G$ ), contradicting the fact that $G$ does not have a $V_{8}$-minor.

In [12] also the following was shown (we thank Andries Brouwer for communicating the proof below to us):

Theorem $13.3 \lambda\left(V_{8}\right)=4$.

Proof The inequality $\lambda\left(V_{8}\right) \leq 4$ follows from the fact that for any vertex $v$ of $V_{8}$, the graph $V_{8}-v$ is planar. Hence $\lambda\left(V_{8}\right) \leq \lambda\left(V_{8}-v\right)+1 \leq 4$ by Theorem 13.1. 
We next show $\lambda\left(V_{8}\right) \geq 4$. Represent $V_{8}$ as the graph $G$ with vertex set $V=\{0, \ldots, 7\}$. Let $M=\left(m_{i, j}\right)$ be the $8 \times 8$ matrix defined by

$$
m_{i, j}= \begin{cases}1 & \text { if } i=j, \\ -\sqrt{2} & \text { if }|i-j|=1 \text { or } 7 \\ -1 & \text { if }|i-j|=4 \\ =0 & \text { otherwise, }\end{cases}
$$

where we assume that the rows and columns are labelled $0, \ldots, 7$.

One can show that $M$ has rank at most 4 as follows. For $\alpha=\frac{1}{4} \pi \mathrm{i}$ and $\alpha=\frac{1}{2} \pi \mathrm{i}$, let $x^{\alpha}$ be the vector in $\mathbb{C}^{8}$ defined by

$$
x_{j}^{\alpha}:=e^{\alpha j}
$$

for $j=0, \ldots, 7$. These two vectors are linearly independent and both satisfy $M x^{\alpha}=0$. Indeed, by symmetry it suffices to show that for both choices of $\alpha$ one has $\left(M x^{\alpha}\right)_{0}=0$. Now

$$
\left(M x^{\alpha}\right)_{0}=x_{0}^{\alpha}-x_{-1}^{\alpha} \sqrt{2}-x_{1}^{\alpha} \sqrt{2}-x_{4}^{\alpha}=1-e^{-\alpha} \sqrt{2}-e^{\alpha} \sqrt{2}-e^{4 \alpha}
$$

taking subscripts mod 8. If $\alpha=\frac{1}{4} \pi \mathrm{i}$, then $e^{\alpha}+e^{-\alpha}=2 \cos \frac{1}{4} \pi=\sqrt{2}$, while $e^{4 \alpha}=\cos \pi+\mathrm{i} \sin \pi=-1$, and hence $\left(M x^{\alpha}\right)_{0}=0$. If $\alpha=\frac{1}{2} \mathrm{i} \pi$, then $e^{\alpha}+e^{-\alpha}=$ $2 \cos \frac{1}{2} \pi=0$, while $e^{4 \alpha}=\cos 2 \pi+\mathrm{i} \sin 2 \pi=1$, and hence again $\left(M x^{\alpha}\right)_{0}=0$. Since the real and imaginary parts of the two vectors $x^{\alpha}$ give four vectors linearly independent over $\mathbb{R}$, we know that $\operatorname{corank}(M) \geq 4$.

Let $X$ be the kernel (null space) of $M$. We show that $X$ is representative for $G$. Choose a nonzero $x \in X$. So

$$
x_{j}=x_{j-1} \sqrt{2}+x_{j+1} \sqrt{2}+x_{j+4} .
$$

Let $W:=\operatorname{supp}^{+}(x)$. Then $W \neq \emptyset$, since otherwise for any $j$ with $x_{j+1}<0$, the value of $x_{j}$ would be strictly smaller than $x_{j+1}$ by (64).

Assume that $W$ induces a disconnected subgraph of $V_{8}$. Let $U:=V \backslash W$, and let $K_{1}$ and $K_{2}$ be two of the components of $G \mid W$. Then $\left|K_{i}\right| \geq 2$, since otherwise $K_{i}$ would consist of one vertex, contradicting (64). So $|U| \leq 4$. Since $V_{8}$ is 3-connected, since each cutset of size 3 consists of the set of vertices adjacent to one vertex, and since $U$ separates $K_{1}$ and $K_{2}$, it follows that $|U|=4$, and that the subgraph induced by $W$ consists of two disjoint edges.

Now for each edge $e=\{j, j+1\}$ of $V_{8}$, each other edge $e^{\prime}$ of $V_{8}$ disjoint from $e$ contains at least one vertex that is adjacent to at least one vertex in $e$. It follows that $W=\{1,3,5,7\}$ or $W=\{0,2,4,6\}$. Then (64) implies that $x_{j} \leq x_{j+4}$ for each $j \in W$, and hence $x_{j}=x_{j+4}$ for each $j \in W$. But then $x_{j}=0$ for each $j \in U$, contradicting the fact that $\operatorname{supp}^{-}(x) \neq \emptyset$.

Thus we have the following theorem: 
Theorem 13.4 Let $G$ be a graph. Then $\lambda(G) \leq 3$ if and only if $G$ arises by taking clique sums and subgraphs from planar graphs; that is, if and only if $G$ has no $K_{5}$ - or $V_{8}$-minor.

Proof Directly from Theorems 5.2, 6.2, 13.1, 13.2, and 13.3.

\section{A Borsuk theorem for antipodal links}

We next come to studying $\mu(G) \leq 4$ and $\lambda(G) \leq 4$. The following Borsuktype theorem on the existence of certain antipodal links is essential in the proof. This theorem, for general dimension, is proved in [15].

Let $P$ be a convex polytope in $\mathbb{R}^{n}$. We say that two faces $F$ and $F^{\prime}$ are antipodal if there exists a nonzero vector $c$ in $\mathbb{R}^{n}$ such that the linear function $c^{T} x$ is maximized by every point of $F$ and minimized by every point of $F^{\prime}$. Let $(P)_{1}$ denote the 1-skeleton of $P$. For any face $F$ of $P$, let $\partial F$ be its boundary.

Theorem 14.1 Let $P$ be a full-dimensional convex polytope in $\mathbb{R}^{5}$ and let $\phi$ be an embedding of $(P)_{1}$ into $\mathbb{R}^{3}$. Then there exists a pair of antipodal 2-faces $F$ and $F^{\prime}$ such that $\phi(\partial F)$ and $\phi\left(\partial F^{\prime}\right)$ are linked.

In [15] this is derived (for general dimension) from a Borsuk-type theorem on the existence of antipodal intersections, extending a result of Bajmóczy and Bárány [2] slightly. A direct proof of Theorem 14.1 can be sketched as follows. First:

We can assume that if $F$ and $F^{\prime}$ are antipodal 2-faces of $P$, then $F-F$ and $F^{\prime}-F^{\prime}$ do not have any nonzero vector in common.

This can be shown by applying a small projective perturbation to $P$.

For any two disjoint closed curves $C$ and $C^{\prime}$ in $\mathbb{R}^{3}$, let $\operatorname{lk}\left(C, C^{\prime}\right)$ denote their linking number, which is the number mod 2 of crossings in any link diagram where $C$ is over $C^{\prime}$. (This is a topological invariant.) Then:

There exists an embedding $\psi:(P)_{1} \rightarrow \mathbb{R}^{3}$ with the property that there is exactly one pair of antipodal 2-faces $F, F^{\prime}$ for which $\operatorname{lk}\left(\psi(\partial F), \psi\left(\partial F^{\prime}\right)\right)=1$.

Indeed, we can assume that by maximizing the last coordinate $x_{5}$ we obtain some 2-face $F_{0}$ and by minimizing $x_{5}$ we obtain some 2-face $F_{0}^{\prime}$ antipodal to $F_{0}$. Moreover, we can assume that $(0,0,0,0,1)$ belongs to the relative interior of $F_{0}$ and that $(0,0,0,0,-1)$ belongs to the relative interior of $F_{0}^{\prime}$. For any vector $x=\left(x_{1}, \ldots, x_{5}\right)$ in $\mathbb{R}^{5}$, let $\tilde{x}:=\left(x_{1}, \ldots, x_{4}\right)$. Then define $\psi:(P)_{1} \rightarrow S^{3}$ by:

$$
\psi(x):=\frac{\tilde{x}}{\|\tilde{x}\|} .
$$


We can assume that $\psi$ is an embedding of $(P)_{1}$ into $S^{3}$ (by moving $P$ slightly). Then $F_{0}, F_{0}^{\prime}$ is the only pair of antipodal 2 -faces $F, F^{\prime}$ for which we have $\operatorname{lk}\left(\psi(\partial F), \psi\left(\partial F^{\prime}\right)\right)=1$.

Finally:

We can deform $\psi$ to $\phi$ while only edges are moved through each other; at each such operation, the quantity $\sum_{F, F^{\prime}} \mathrm{lk}\left(\partial F, \partial F^{\prime}\right)$ remains invariant.

This follows from the fact that for any two edges $e, e^{\prime}$ of $(P)_{1}$, the number of pairs of antipodal 2-faces $F, F^{\prime}$ with $e \subset \partial F$ and $e^{\prime} \subset \partial F^{\prime}$, is even.

\section{Characterizing $\mu(G) \leq 4$}

We first give a brief introduction to the work of Robertson, Seymour, and Thomas on linklessly embeddable graphs. An embedding of a graph $G$ into $\mathbb{R}^{3}$ is called linkless if any two disjoint circuits in $G$ have unlinked images in $\mathbb{R}^{3}$. A graph $G$ is linklessly embeddable (in $\mathbb{R}^{3}$ ) if it has a linkless embedding in $\mathbb{R}^{3}$.

There are a number of equivalent characterizations of linklessly embeddable graphs. Call an embedding of $G$ flat if for each circuit $C$ in $G$ there is a disk $D$ (a 'panel') disjoint from (the embedding of) $G$ and having boundary equal to $C$. Clearly, each flat embedding is linkless, but the reverse does not hold. (For instance, if $G$ is just a circuit $C$, then any embedding of $G$ is linkless, but only the unknotted embeddings are flat.) However, if $G$ has a linkless embedding, it also has a flat embedding. So the collections of linklessly embeddable graphs and of flatly embeddable graphs are the same. This was shown by Robertson, Seymour, and Thomas [19], as a byproduct of a proof of an even deeper forbidden-minor characterization of linklessly embeddable graphs.

To understand this forbidden-minor characterization, it is important to note that the class of linklessly embeddable graphs is closed under the Y $\Delta$ and $\Delta Y$-operations. It implies that also the class of forbidden minors for linkless embeddability is closed under applying $\mathrm{Y} \Delta$ and $\Delta \mathrm{Y}$. Now Robertson, Seymour, and Thomas [19] showed:

the Petersen family is the collection of forbidden minors for

linkless embeddability.

Here the Petersen family is the class of graphs arising from the Petersen graph by any series of $\Delta Y$ - and $Y \Delta$-operations. The Petersen family consists of seven graphs, and includes the graph $K_{6}$ (see Figure 2).

It turns out not to be difficult to prove that $\mu(G)=5$ for each graph in the Petersen family. In fact, by result (51) of Bacher and Colin de Verdière [1], the class of graphs $G$ with $\mu(G)=5$ is closed under $\Delta Y$ and $Y \Delta$. Since moreover $\mu\left(K_{6}\right)=5$, we know $\mu(G)=5$ for each graph $G$ in the Petersen family. 

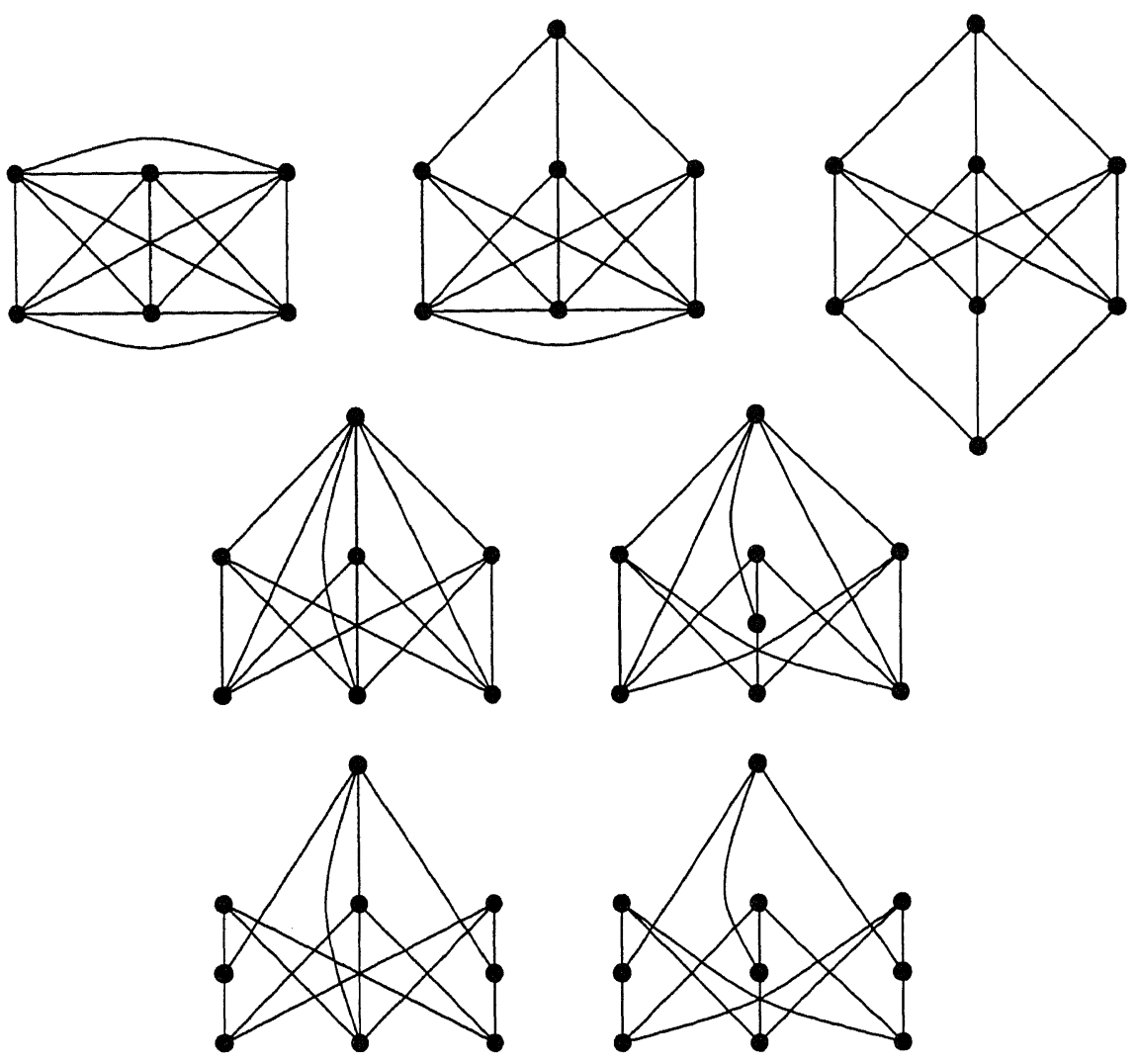

Figure 2: The Petersen family

So with the forbidden minor characterization of Robertson, Seymour, and Thomas [19], we know that if $\mu(G) \leq 4$ then $G$ is linklessly embeddable. The reverse implication was conjectured by Robertson, Seymour, and Thomas [17] and proved in [15], and thus we have:

Theorem 15.1 $\mu(G) \leq 4$ if and only if $G$ is linklessly embeddable; that is, if and only if $G$ does not have a minor in the Petersen family.

We will not give the full proof of this here, but rather give an indication of the proof by showing that $\lambda(G) \leq 4$ for each linklessly embeddable graph $G$. The proof that also $\mu(G) \leq 4$ for linklessly embeddable graphs $G$ is similar, but requires a few more technicalities, and we do not give it in this paper. 


\section{Towards characterizing $\lambda(G) \leq 4$}

We do not know a complete characterization of the class of graphs $G$ satisfying $\lambda(G) \leq 4$. However, we have ([15]):

Theorem 16.1 If $G$ is linklessly embeddable, then $\lambda(G) \leq 4$.

Proof Let $G$ be linklessly embedded in $\mathbb{R}^{3}$, and suppose that $\lambda(G) \geq 5$. Then there is a 5 -dimensional subspace $L$ of $\mathbb{R}^{V}$ such that $G \mid \operatorname{supp}^{+}(x)$ is nonempty and connected for each nonzero $x \in L$.

Call two elements $x$ and $x^{\prime}$ of $L$ equivalent if $\operatorname{supp}^{+}(x)=\operatorname{supp}^{+}\left(x^{\prime}\right)$ and $\operatorname{supp}^{-}(x)=\operatorname{supp}^{-}\left(x^{\prime}\right)$. The equivalence classes decompose $L$ into a centrally symmetric complex $\mathcal{P}$ of pointed polyhedral cones. Choose a sufficiently dense set of vectors of unit length from every cone in $\mathcal{P}$, in a centrally symmetric fashion, and let $P$ be the convex hull of these vectors. Then $P$ is a 5 -dimensional centrally symmetric convex polytope such that every face of $P$ is contained in a cone of $\mathcal{P}$.

We define an embedding $\phi$ of $(P)_{1}$ in $\mathbb{R}^{3}$. For each vertex $v$ of $P$, we choose a vertex $v^{\prime}$ of $G$ in $\operatorname{supp}^{+}(v)$, and we let $\phi(x)$ be a point in $\mathbb{R}^{3}$ very near $v^{\prime}$. For each edge $e=u v$ of $P$, we choose a path $e^{\prime}$ connecting $u^{\prime}$ and $v^{\prime}$ in $G \mid \operatorname{supp}^{+}(x)$, where $x$ is an interior point of $e$. (By our construction, $\operatorname{supp}^{+}(x)$ is independent of the choice of $x$, and contains both supp ${ }^{+}(u)$ and $\operatorname{supp}^{+}(v)$.) Then we map $e$ onto a Jordan curve connecting $\phi(u)$ and $\phi(v)$ very near $e^{\prime}$. Clearly we can choose the images of the vertices and edges so that this map $\phi$ is one-to-one.

Then by Theorem 14.1, $P$ has two antipodal 2 -faces $F$ and $F^{\prime}$ such that the images of their boundaries are linked. Since $P$ is centrally symmetric, there is a facet $D$ of $P$ such that $F \subseteq \bar{D}$ and $F^{\prime} \subseteq-\bar{D}$. Let $y$ be a vector in the interior of $D$. Then the images of $\partial F$ and $\partial F^{\prime}$ are very near subgraphs spanned by $\operatorname{supp}^{+}(y)$ and $\operatorname{supp}^{-}(y)$, respectively, and hence some circuit of $G$ spanned by $\operatorname{supp}^{+}(y)$ must be linked with some circuit in $\operatorname{supp}^{-}(y)$, a contradiction.

Corollary 16.2 If $G$ is obtained from linklessly embedded graphs by taking clique sums and subgraphs, then $\lambda(G) \leq 4$.

Proof Directly from Theorems 6.2 and 16.1.

By Theorem 5.2, $G=K_{6}$ is a forbidden minor for the class of graphs $G$ with $\lambda(G) \leq 4$. Any other graph $G$ in the Petersen family of graphs however satisfies $\lambda(G) \leq 4$, since:

Theorem 16.3 Let $G$ be in the Petersen family with $G \neq K_{6}$. Then $G$ is obtainable by taking clique sums and subgraphs from $K_{5}$. 
Proof Inspection of the Petersen family (Figure 2) shows that $G$ is either a subgraph of the graph obtained from $K_{7}$ by deleting the edges of a triangle, and this graph is a clique sum of three $K_{5}$ 's, or $G$ arises from such a subgraph by one or more $\Delta Y$-transformations, that is, it is a subgraph of a clique sum with $K_{4}$ 's.

This immediately implies that $\lambda(G) \leq 4$ for each graph $G \neq K_{6}$ in the Petersen family. Moreover, it follows that each such graph is obtainable by taking clique sums and subgraphs from linklessly embeddable graphs.

Note that the graph $G$ obtained from $V_{8}$ by adding a new vertex adjacent to all vertices of $V_{8}$, cannot be obtained from linklessly embeddable graphs by taking clique sums and subgraphs; but $G$ does not have a $K_{6}$-minor. In fact, it satisfies $\lambda(G)=5$. However it is not minor-minimal for the property $\lambda(G) \geq 5$.

Let $V_{9}^{\prime}$ arise from $V_{8}$ by adding an extra vertex $v_{0}$, adjacent to $v_{2}, v_{4}, v_{6}, v_{7}$, $v_{8}$ (see Figure 3). Similarly, let $V_{9}^{\prime \prime}$ arise from $V_{8}$ by adding an extra vertex $v_{0}$

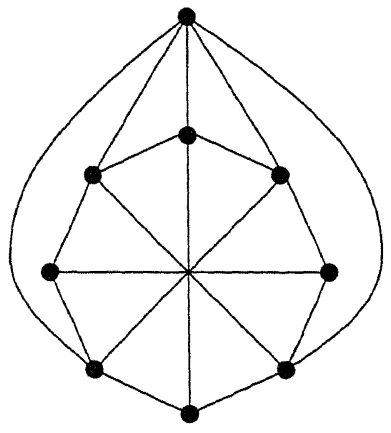

Figure 3: The graph $V_{9}^{\prime}$

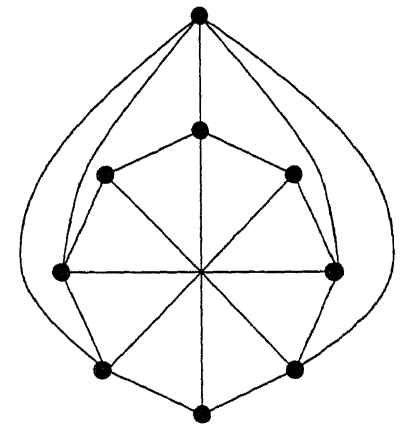

Figure 4: The graph $V_{9}^{\prime \prime}$

adjacent to $v_{2}, v_{3}, v_{5}, v_{7}, v_{8}$ (see Figure 4). It is shown in [12] that $V_{9}^{\prime}$ and $V_{9}^{\prime \prime}$ are minor-minimal graphs $G$ with $\lambda(G) \geq 5$.

The graphs $V_{9}^{\prime}$ and $V_{9}^{\prime \prime}$ are also minor-minimal graphs not obtainable from linklessly embeddable graphs by taking clique sums and subgraphs. This can be seen as follows. Since $\lambda\left(V_{9}^{\prime}\right)=\lambda\left(V_{9}^{\prime \prime}\right)=5$, it follows from Corollary 16.2 that these two graphs indeed are not obtainable in such a way. Moreover, to see that they are minor-minimal, observe that deleting or contracting any edge of $V_{9}^{\prime}$ or $V_{9}^{\prime \prime}$, produces a graph that has a vertex whose deletion makes the graph a clique sum of planar graphs.

Since the class of graphs $G$ with $\lambda(G) \leq 4$ is closed under taking $\Delta \mathrm{Y}$ operations (not under $\mathrm{Y} \Delta$ ), we can obtain other graphs with $\lambda(G) \geq 5$ by applying a $Y \Delta$ operation to $V_{9}^{\prime}$ or $V_{9}^{\prime \prime}$. Any of them contains a $K_{6}$-minor, except if we apply $Y \Delta$ to vertex $v_{1}$ (or equivalently, to $v_{5}$ ) of $V_{9}^{\prime}$. 


\section{An extension to oriented matroids}

It turns out that the results described above for $\lambda(G)$ can be extended to oriented matroids, as is shown in [7]. Before describing this, we first give the definition of and a little further background on oriented matroids (see Björner, Las Vergnas, Sturmfels, White, and Ziegler [3] for more information).

It is convenient to introduce, for any ordered pair $x=(a, b)$, the notation $x^{+}:=a$ and $x^{-}:=b$, and $-x=(b, a)$.

An oriented matroid $(V, X)$ consists of a finite set $V$ and a collection $X$ of ordered pairs $x=\left(x^{+}, x^{-}\right)$of subsets of $V$ such that:

(i) for each $x \in X, x^{+} \cap x^{-}=\emptyset$;

(ii) $0:=(\emptyset, \emptyset) \in X$

(iii) if $x \in X$ then $-x \in X$;

(iv) if $x, y \in X$, then $x \cdot y \in X$ where $x \cdot y$ is defined by $x \cdot y:=\left(x^{+} \cup\left(y^{+} \backslash x^{-}\right), x^{-} \cup\left(y^{-} \backslash x^{+}\right)\right)$;

(v) if $x, y \in X$ and $u \in x^{+} \cap y^{-}$, then there exists a $z \in X$ such that $u \notin z^{+} \cup z^{-},\left(x^{+} \backslash y^{-}\right) \cup\left(y^{+} \backslash x^{-}\right) \subseteq z^{+} \subseteq x^{+} \cup y^{+}$, and $\left(x^{-} \backslash y^{+}\right) \cup\left(y^{-} \backslash x^{+}\right) \subseteq z^{-} \subseteq x^{-} \cup y^{-}$.

The elements of $X$ are called the vectors of the oriented matroid. ( 0 is the zero.) Any linear subspace $Y$ of $\mathbb{R}^{V}$ gives an oriented matroid $(V, X)$, by taking

$$
\left.X:=\left\{\operatorname{supp}^{+}(x), \operatorname{supp}^{-}(x)\right) \mid x \in Y\right\} .
$$

For any oriented matroid $M=(V, X)$, the minimal nonempty subsets of $\left\{x^{+} \cup x^{-} \mid x \in X\right\}$ form the circuit collection of a matroid, again denoted by $M$. Thus matroid terminology applies to oriented matroids, and we can speak of the rank $\operatorname{rank}(M)$ of an oriented matroid $M$ : it is the maximum size of a subset of $V$ not containing any circuit as a subset. The corank corank $(M)$ of $M$ is equal to $|V|-\operatorname{rank}(M)$. It is not difficult to prove that if $M$ is given by (71), then

$$
\operatorname{corank}(M)=\operatorname{dim}(Y) \text {. }
$$

Now the graph parameter $\lambda^{\prime}(G)$ is defined as follows. Let $G=(V, E)$ be an undirected graph. An oriented matroid $M=(V, X)$ is called representative for $G$ if

for each nonzero $x \in X, x^{+}$is nonempty and induces a connected subgraph of $G$.

Then $\lambda^{\prime}(G)$ is the largest corank of an oriented matroid representative for $G$.

From (72) one derives that for each graph $G$ :

$$
\lambda(G) \leq \lambda^{\prime}(G) .
$$


One of the consequences of the results described below is that there are no graphs $G$ with $\lambda(G) \leq 3$ and $\lambda(G)<\lambda^{\prime}(G)$. In fact, we do not know any graph $G$ with strict inequality in (74).

Any result we know for $\lambda(G)$, also holds for $\lambda^{\prime}(G)$. First of all, $\lambda^{\prime}(G)$ is minor-monotone:

if $G$ is a minor of $H$ then $\lambda^{\prime}(G) \leq \lambda^{\prime}(H)$.

Moreover one has:

$$
\lambda^{\prime}\left(K_{n}\right)=n-1 .
$$

So again Hadwiger's conjecture implies the conjecture that $\gamma(G) \leq \lambda^{\prime}(G)+1$ for each graph $G$, where $\gamma(G)$ is the colouring number of $G$.

Moreover:

For any graph $G$ and vertex $v$ of $G$ one has $\lambda^{\prime}(G-v) \geq$ $\lambda^{\prime}(G)-1$.

Again for each $t \geq 1$ the class of graphs $G$ with $\lambda(G) \leq t$ is closed under taking clique sums, since:

If $G$ has at least one edge and is a clique sum of $G_{1}$ and $G_{2}$, then $\lambda^{\prime}(G)=\max \left\{\lambda^{\prime}\left(G_{1}\right), \lambda^{\prime}\left(G_{2}\right)\right\}$.

This directly implies characterizations of those graphs $G$ satisfying $\lambda^{\prime}(G) \leq 1$ and $\lambda^{\prime}(G) \leq 2$ :

$$
\lambda^{\prime}(G) \leq 1 \text { if and only if } G \text { is a forest, }
$$

and

$\lambda^{\prime}(G) \leq 2$ if and only if $G$ is a series-parallel graph.

Moreover, it can be proved that

a graph $G$ satisfies $\lambda^{\prime}(G) \leq 3$ if and only if $G$ can be obtained

from planar graphs by taking clique sums and subgraphs.

Recently, Rudi Pendavingh showed:

if $G$ is obtainable from linklessly embeddable graphs by taking subgraphs and clique sums, then $\lambda^{\prime}(G) \leq 4$.

\section{The related graph invariant $\kappa(G)$}

We finally describe a graph invariant related to $\lambda(G)$ (introduced in [12]), for which the set of forbidden minors can be precisely characterized. For any 
connected graph $G=(V, E)$, define $\kappa(G)$ to be the largest $d$ for which there exists a function $\phi: V \rightarrow \mathbb{R}^{d}$ such that:

(i) $\phi(V)$ affinely spans a $d$-dimensional affine space;

(ii) for each affine halfspace $H$ of $\mathbb{R}^{d}, \phi^{-1}(H)$ induces a connected subgraph of $G$ (possibly empty).

(An affine halfspace is a set of the form $\left\{x \mid c^{T} x>\delta\right\}$ for some nonzero vector $c$.) Note that such a function $\phi$ does not exist for disconnected graphs; so $\kappa(G)$ is undefined if $G$ is disconnected.

Observe that if $G$ is the 1-skeleton of a full-dimensional polytope in $\mathbb{R}^{d}$, then $\kappa(G) \geq d$, as the polytope gives the embedding in $\mathbb{R}^{d}$.

By similar arguments as used in the proof of Theorem 3.1 one shows that if $H$ is a connected minor of $G$ then $\kappa(H) \leq \kappa(G)$. So again for each $d$ there is a finite collection of forbidden minors for the collection of graphs satisfying $\kappa(G) \leq d$. This collection of graphs is equal to $\left\{K_{d+2}\right\}$, as is shown in the next theorem.

First observe that

$$
\kappa(G) \leq \lambda(G)
$$

holds for each connected graph $G$, since if $\phi: V \rightarrow \mathbb{R}^{d}$ satisfies (83), then we may assume that the origin belongs to the interior of the convex hull of $\phi(V)$. But then trivially $\phi$ is representative for $G$.

Basic in the characterization is the following observation (Grünbaum and Motzkin [9], Grünbaum [8]):

Theorem 18.1 If $G$ is the 1-skeleton of a $d$-dimensional polytope $P$, then $G$ has a $K_{d+1}$-minor.

Proof By induction on $d$, the case $d=0$ being trivial. If $d>0$, let $F$ be a facet of $P$. By the induction hypothesis, the 1-skeleton of $F$ can be contracted to $K_{d}$. Moreover, the vertices of $P$ not on $F$ induce a connected subgraph of $G$, and hence can be contracted to one vertex. This yields a contraction of $G$ to $K_{d+1}$, as each vertex of $F$ is adjacent to at least one vertex of $P$ not on $F$.

This gives:

Theorem 18.2 For each connected graph $G$ and each $d, \kappa(G) \geq d$ if and only if $G$ has a $K_{d+1}$-minor.

Proof Sufficiency. One has $\kappa\left(K_{d+1}\right)=d$ since the vertices of a simplex in $\mathbb{R}^{d}$ give a function $\phi$ satisfying (83). So if $G$ has a $K_{d+1}$-minor, then $\kappa(G) \geq d$. 
Necessity. Let $G=(V, E)$ be a connected graph and let $d:=\kappa(G)$, such that for each proper connected minor $H$ one has $\kappa(H)<d$. By Theorem 18.1 it suffices to show that $G$ is the 1-skeleton of a $d$-dimensional polytope.

Let $\phi: V \rightarrow \mathbb{R}^{d}$ satisfy (83). Let $P$ be the convex hull of $\phi(V)$. So $P$ is a $d$-dimensional polytope in $\mathbb{R}^{d}$. We show that $G$ is the 1-skeleton of $P$.

First observe that for each vertex $x$ of $P$, the set $\phi^{-1}(x)$ induces a connected subgraph of $G$, as it is equal to $\phi^{-1}(H)$ for some affine halfspace $H$ of $\mathbb{R}^{d}$. Hence if $\phi^{-1}(x)$ consists of more than one vertex of $G$, then we can contract this subgraph to one vertex, contradicting the minimality of $G$.

Similarly, for each edge $x y$ of $P$, the set $\phi^{-1}(x y)$ induces a connected subgraph of $G$. Hence it contains a path from $\phi^{-1}(x)$ to $\phi^{-1}(y)$.

As this is true for each edge, $G$ contains a subdivision of the 1-skeleton of $P$ as a subgraph. By the minimality of $G$ this implies that $G$ is equal to the 1-skeleton of $P$.

So Hadwiger's conjecture is equivalent to $\gamma(G) \leq \kappa(G)+1$ for each connected graph $G$.

\section{Acknowledgements}

We thank the referee for several suggestions improving the presentation.

\section{References}

[1] R. Bacher \& Y. Colin de Verdière, Multiplicités des valeurs propres et transformations étoile-triangle des graphes, Bulletin de la Société Mathématique de France, 123 (1995), 101-117.

[2] E. G. Bajmóczy \& I. Bárány, On a common generalization of Borsuk's and Radon's theorem, Acta Mathematica Academiae Scientiarum Hungaricae, 34 (1979), 347-350.

[3] A. Björner, M. Las Vergnas, B. Sturmfels, N. White \& G. Ziegler, Oriented Matroids, Cambridge University Press, Cambridge (1993).

[4] S. Y. Cheng, Eigenfunctions and nodal sets, Commentarii Mathematici Helvetici, 51 (1976), 43-55.

[5] Y. Colin de Verdière, Sur un nouvel invariant des graphes et un critère de planarité, Journal of Combinatorial Theory, Series B, 50 (1990), 11-21.

[6] Y. Colin de Verdière, On a new graph invariant and a criterion for planarity, in Graph Structure Theory (Proceedings of the AMS-IMS-SIAM Joint Summer Research Conference on Graph Minors, Seattle, 1991) (eds. N. Robertson \& P. Seymour), Contemporary Mathematics, 147, American Mathematical Society, Providence, Rhode Island (1993), 137-147. 
[7] J. Edmonds, M. Laurent \& A. Schrijver, A minor-monotone graph parameter based on oriented matroids, Discrete Mathematics, in press.

[8] B. Grünbaum, On the facial structure of convex polytopes, Bulletin of the American Mathematical Society, 71 (1965), 559-560.

[9] B. Grünbaum \& T S. Motzkin, On polyhedral graphs, in Convexity (ed. V. Klee), Proceedings of Symposia in Pure Mathematics, 7, American Mathematical Society, Providence, Rhode Island (1963), pp. 285-290.

[10] H. van der Holst, A short proof of the planarity characterization of Colin de Verdière, Journal of Combinatorial Theory, Series B, 65 (1995), 269272 .

[11] H. van der Holst, Topological and Spectral Graph Characterizations, Ph.D. Thesis, University of Amsterdam, 1996.

[12] H. van der Holst, M. Laurent \& A. Schrijver, On a minor-monotone graph invariant, Journal of Combinatorial Theory, Series B, 65 (1995), 291-304.

[13] H. van der Holst, L. Lovász \& A. Schrijver, On the invariance of Colin de Verdière's graph parameter under clique sums, Linear Algebra and its Applications, 226 (1995), 509-517.

[14] A. Kotlov, L. Lovász \& S. Vempala, The Colin de Verdière number and sphere representations of a graph, preprint, 1996.

[15] L. Lovász \& A. Schrijver, A Borsuk theorem for antipodal links and a spectral characterization of linklessly embeddable graphs, Proceedings of the American Mathematical Society, in press.

[16] N. Robertson \& P. D. Seymour, Graph minors. XX. Wagner's conjecture, preprint, 1988.

[17] N. Robertson, P. D. Seymour \& R. Thomas, A survey of linkless embeddings, in Graph Structure Theory (Proceedings of the AMS-IMS-SIAM Joint Summer Research Conference on Graph Minors, Seattle, 1991) (eds. N. Robertson \& P. Seymour), Contemporary Mathematics, 147, American Mathematical Society, Providence, Rhode Island (1993), pp. 125-136.

[18] N. Robertson, P. Seymour \& R. Thomas, Hadwiger's conjecture for $K_{6}{ }^{-}$ free graphs, Combinatorica, 13 (1993), 279-361.

[19] N. Robertson, P. Seymour \& R. Thomas, Sachs' linkless embedding conjecture, Journal of Combinatorial Theory, Series B, 64 (1995), 185-227.

[20] K. Wagner, Über eine Eigenschaft der ebene Komplexe, Mathematische Annalen, 114 (1937), 570-590. 
CWI

Kruislaan 413

1098 SJ Amsterdam

The Netherlands

and

Department of Mathematics

University of Amsterdam

Plantage Muidergracht 24

1018 TV Amsterdam

The Netherlands

lex@cwi.nl 